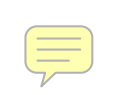

\title{
$\triangle$-COHERENT PAIRS AND ORTHOGONAL POLYNOMIALS OF A DISCRETE VARIABLE
}

\author{
I. AREA ${ }^{\mathrm{a}, *}$, E. GODOY ${ }^{\mathrm{a}, \dagger}$ and F. MARCELLÁN ${ }^{\mathrm{b},+}$ \\ ${ }^{a}$ Departamento de Matemática Aplicada, E.T.S.I. Industriales y Minas, Universidad de Vigo, \\ Campus Lagoas Marcosende, 36200 Vigo, Spain; ${ }^{b}$ Departamento de Matemáticas, Escuela \\ Politécnica Superior, Universidad Carlos III, Avenida de la Universidad, 30, 28911 \\ Leganés Madrid, Spain
}

In this paper we define the concept of $\Delta$-coherent pair of linear functionals. We prove that if $\left(u_{0}, u_{1}\right)$ is a $\Delta$-coherent pair of linear functionals then at least one of them must be a classical discrete linear functional under certain conditions. Examples related to Meixner and Hahn linear functionals are given.

Keywords: Orthogonal polynomials of a discrete variable; Linear functionals; $\Delta$-coherent pairs

1991 Mathematics Subject Classification: 33C25; 42C05

\section{INTRODUCTION}

The concepts of coherent pair and symmetric coherent pair have been introduced by A. Iserles et al. in Ref. [9] in the framework of the study of orthogonal polynomials associated with the Sobolev inner product

$$
\langle f, g\rangle_{S}=\int_{\mathbb{R}} f g \mathrm{~d} \mu_{0}+\lambda \int_{\mathbb{R}} f^{\prime} g^{\prime} \mathrm{d} \mu_{1},
$$

where $\mu_{0}$ and $\mu_{1}$ are non-atomic positive Borel measures on the real line such that

$$
\left|\int_{\mathbb{R}} x^{k} \mathrm{~d} \mu_{i}(x)\right|<\infty, \quad k \geq 0, \quad i=0,1 .
$$

\footnotetext{
* Corresponding author. E-mail: area@dma.uvigo.es

† E-mail: egodoy@dma.uvigo.es

E-mail: pacomarc@ing.uc3m.es
} 
In fact, coherence means that a relation between the MOPS (monic orthogonal polynomial sequence) $\left\{\mathrm{P}_{n}(x)\right\}_{n}$ and $\left\{\mathrm{T}_{n}(x)\right\}_{n}$, associated with the measures $\mu_{0}$ and $\mu_{1}$ respectively,

$$
\mathrm{T}_{n}(x)=\frac{\mathrm{P}_{n+1}^{\prime}(x)}{n+1}-\sigma_{n} \frac{\mathrm{P}_{n}^{\prime}(x)}{n}, \quad n \geq 1,
$$

where $\left\{\sigma_{n}\right\}_{n}$ is a sequence of non-zero complex numbers, is satisfied.

The description of the measures satisfying the coherence condition was an open problem in Ref. [9]. A first result is given in Ref. [15] where the complete set of coherence pairs when one of the measures is a classical one (Hermite, Laguerre, Jacobi) is described. Later on, in Ref. [16] it is proved that both measures involved in a coherent pair must be semiclassical.

Finally, in Ref. [20] H. G. Meijer gave the complete classification of the coherent pairs of measures which is, essentially, the same stated in Ref. [15]. As a conclusion, the Meijer's result shows that coherent pairs of measures constitute a restrictive class, helpful in order to obtain properties of polynomials orthogonal with respect to Sobolev inner products $[14,19,22,23]$.

The aim of this paper is to analyze the extension of the above definition of coherent pairs (based in the use of the derivative operator) to the situation when the forward difference operator $\Delta$ is considered. Thus, orthogonal polynomials of a discrete variable [21] appear in a natural way. Our main result states that if $\left(u_{0}, u_{1}\right)$ is a $\Delta$-coherent pair of linear functionals, one of them must be a classical discrete linear functional. In this situation, the support of the functional can be finite (Kravchuk, Hahn) or infinite (Meixner, Charlier) and the extension of the Meijer's result involves more than a merely formal analogy. First, the concept of weakly quasi-definite linear functional (including both finite and infinite supporting sets for the functional) is introduced in order to cover both situations. Second, using a limit process we can recover the Meijer's classification from the complete description of the $\Delta$-coherent pairs which we have already stated in Ref. [5]. Third, we compare the complexity of the analysis for the $\Delta$ operator with the derivative operator, because the situation, in our case, is more interesting taking into account that not only quasi-definite linear functionals are involved.

The outline of the paper is as follows: in Section 2 we give the basic definitions and results which will be helpful in the following sections. In Section 3 we introduce the concept of $\Delta$-coherent pair of linear functionals, and we prove that if $\left(u_{0}, u_{1}\right)$ is a $\Delta$-coherent pair, both $u_{0}$ and $u_{1}$ are semiclassical discrete linear functionals. In Section 4 we prove that if $\left(u_{0}, u_{1}\right)$ is a $\Delta$-coherent pair of linear functionals, at least one of them must be a classical discrete linear functional, where $u_{0}$ and $u_{1}$ are required to have a restriction on their orders. Finally, in Section 5 we present two examples of $\Delta$-coherent pair of linear functionals related to the Meixner and the Hahn linear functional, as examples of quasi-definite and weakly quasi-definite linear functionals, respectively.

\section{NOTATIONS AND BASIC RESULTS}

Let $\mathbb{P}$ be the linear space of polynomials with complex coefficients and let $\mathbb{P}^{\prime}$ be its algebraic dual space. We denote by $\langle u, f\rangle$ the duality bracket for $u \in \mathbb{P}^{\prime}$ and $f \in \mathbb{P}$, and we denote by $(u)_{n}=\left\langle u, x^{n}\right\rangle$, with $n \geq 0$, the canonical moments of $u$.

DEFINITION 2.1 A linear functional $u$ is said to be weakly quasi-definite if there exists $0 \leq M \leq \infty$ such that the principal submatrices $\mathbf{H}_{k}:=\left[(u)_{i+j}\right]_{i, j=0}^{k}$ are nonsingular for 
$0 \leq k \leq M$ and, if $M \neq \infty, \mathbf{H}_{M+1}$ is a singular matrix. $M$ is said to be the order of the linear functional $u$.

Remark 1 Note that when $M=\infty$ this definition coincides with the concept of quasidefinite linear functional given in Ref. [6, p. 16]. In what follows, we shall have in mind this fact despite the notation associated to the order of the linear functional.

Given a weakly quasi-definite linear functional $u$ of order $M$, there exists a family of monic polynomials $\left\{\mathrm{P}_{n}(x)\right\}_{n=0}^{M}$ orthogonal with respect to $u$, i.e. $\mathrm{P}_{n}(x)=x^{n}+$ terms of lower degree, for every $0 \leq n \leq M$, and $\left\langle u, \mathrm{P}_{n} \mathrm{P}_{m}\right\rangle=\Gamma_{n} \delta_{n, m}, \Gamma_{n} \neq 0$, for every $0 \leq n, m \leq M$. Such a sequence will be called monic orthogonal polynomial sequence (MOPS).

Remark 2 Given a weakly quasi-definite linear functional $u$ of order $M<\infty$, it is possible to build a unique finite family of monic polynomials $\left\{\mathrm{P}_{n}(x)\right\}_{n=0}^{M+1}$ such that

$$
\begin{array}{ll}
\left\langle u, x^{m} \mathrm{P}_{n}(x)\right\rangle=0 & 0 \leq m \leq n-1, \quad 1 \leq n \leq M+1, \\
\left\langle u, x^{n} \mathrm{P}_{n}(x)\right\rangle \neq 0 & 0 \leq n \leq M,
\end{array}
$$

and $\left\langle u, x^{M+1} \mathrm{P}_{M+1}(x)\right\rangle=0$. Thus, the sequence of orthogonal polynomials is $\left\{\mathrm{P}_{n}(x)\right\}_{n=0}^{M}$. Note that this sequence of orthogonal polynomials does not generate $\mathbb{P}$ when $M<\infty$.

In the most important occurrences of orthogonal polynomials, the linear functional $u$ satisfies an extra condition [6, p. 13].

DEFINITION 2.2 A linear functional $u$ is called positive-definite if its moments are all real and $\operatorname{det}\left(\mathbf{H}_{k}\right)>0$, for every $k \geq 0$.

DEFINITION 2.3 Given a complex number $c$, the Dirac functional $\delta_{c}$ is defined by $\left\langle\delta_{c}, p(x)\right\rangle:=p(c)$, for every $p \in \mathbb{P}$.

DEFINITION 2.4 Given a linear functional $u$ and a polynomial $p$, we define the linear functional pu as $\langle p u, q\rangle:=\langle u, p q\rangle$, for every $q \in \mathbb{P}$. For each complex number $c$, the linear functional $(x-c){ }^{1} u$ is given by

$$
\left\langle(x-c){ }^{1} u, q\right\rangle:=\left\langle u, \frac{q(x)-q(c)}{x-c}\right\rangle
$$

for every $q \in \mathbb{P}$.

Note that

$$
(x-c){ }^{1}((x-c) u)=u-(u)_{0} \delta_{c}, \quad \text { for every } u \in \mathbb{P}^{\prime},
$$

while $(x-c)\left((x-c){ }^{1} u\right)=u$.

DEFINITION 2.5 The forward and backward difference operators $\Delta$ and $\nabla$ are defined by

$$
\Delta p(x):=p(x+1)-p(x) \quad \nabla p(x):=p(x)-p(x-1),
$$

for every $p \in \mathbb{P}$, respectively. 
Let $p$ and $q$ be two polynomials. Then, we have

$$
\begin{aligned}
& \Delta \nabla=\nabla \Delta, \quad \Delta=\nabla+\Delta \nabla, \quad \Delta p(x)=\nabla p(x+1), \\
& \Delta(p(x) q(x))=q(x) \Delta p(x)+p(x+1) \Delta q(x) .
\end{aligned}
$$

DEFINITION 2.6 For $u \in \mathbb{P}^{\prime}$, the linear functional $\Delta u$ is defined as $\langle\Delta u, p\rangle=-\langle u, \Delta p\rangle$, for every $p \in \mathbb{P}$.

Proposition 2.7 For $u \in \mathbb{P}^{\prime}$ and $p \in \mathbb{P}$, we have $\Delta[p(x) u]=p(x-1) \Delta u+\Delta p(x-1) u$. In particular, we obtain $(x-c) \Delta u=\Delta[(x+1-c) u]-u$.

DEFINITION 2.8 A linear functional $u$ is said to be a classical discrete linear functional if $u$ is weakly quasi-definite and there exist polynomials $\phi$ and $\psi$, with $\operatorname{deg}(\phi) \leq 2$ and $\operatorname{deg}(\psi)=1$, such that

$$
\Delta[\phi(x) u]=\psi(x) u
$$

The corresponding MOPS associated with $u$ is said to be a classical discrete MOPS.

Classical discrete orthogonal polynomials can be characterized by means of the Hahn's property in the following way [see Ref. 7].

PROPOSITION 2.9 Let $\left\{\mathrm{P}_{n}(x)\right\}_{n=0}^{M}$ be the MOPS associated with a weakly quasi-definite linear functional $u$ of order $M \geq 1$. The sequence $\left\{\mathrm{P}_{n}(x)\right\}_{n=0}^{M}$ is a classical discrete MOPS if and only if $\left\{\mathrm{Q}_{n}(x)\right\}_{n=0}^{M} 1$ defined by

$$
\mathrm{Q}_{n}(x):=\frac{\Delta \mathrm{P}_{n+1}(x)}{n+1}, \quad 0 \leq n \leq M-1,
$$

is also a MOPS. Furthermore, if $u$ satisfies $\Delta[\phi(x) u]=\psi(x) u$ then $\left\{\mathrm{Q}_{n}(x)\right\}_{n=0}^{M}{ }^{1}$ is orthogonal with respect to the functional $\breve{u}:=\phi(x) u$.

Note that weakly positive-definite classical discrete functionals are associated with a measure $\varrho(x)$ whose support is a countable set. In such a sense, we shall only consider here orthogonal polynomials of a discrete variable on $[a, b-1]$ with weight $\varrho(x)$,

$$
\sum_{x_{i}=a}^{b} \mathrm{P}_{m}\left(x_{i}\right) \mathrm{P}_{n}\left(x_{i}\right) \varrho\left(x_{i}\right)=d_{n}^{2} \delta_{n, m}, \quad x_{i+1}=x_{i}+1,
$$

provided that the interval $(a, b)$ is contained in $\mathbb{R}$ and the function $\varrho(x)$ satisfies

$$
\Delta(\sigma(x) \varrho(x))=\psi(x) \varrho(x), \quad \sigma(x):=\phi(x)-\psi(x),
$$

with

$$
\left.\sigma(x) \varrho(x) x^{\ell}\right|_{x=a, b}=0, \quad \ell=0,1, \ldots
$$


Thus, classical discrete linear functionals $u$ satisfying (2.2) can be represented as

$$
\langle u, p\rangle=\sum_{x_{i}=a}^{b} p\left(x_{i}\right) \varrho\left(x_{i}\right), \quad \text { for every } p \in \mathbb{P},
$$

where $\varrho(x)$ is a weight function satisfying (2.4) and (2.5). In this situation, classical discrete linear functionals are the corresponding to Hahn, Meixner, Kravchuk and Charlier MOPS [21]. Charlier and Meixner functionals are quasi-definite linear functionals and therefore the corresponding MOPS are infinite sequences. On the other hand, Kravchuk and Hahn linear functionals are weakly quasi-definite linear functionals and the corresponding MOPS are finite. Some applications of these finite families can be found in e.g. [12,21].

In Ref. [11] another systematic study of (positive-definite) orthogonal polynomials of a discrete variable is given from the second order linear difference equation they satisfy. Moreover, a self-contained overview of classical discrete polynomials has been done in Ref. [1], where finite orthogonal sequences are not considered (only quasi-definite functionals are studied).

In the aforementioned classifications $[1,11]$ there appear other families of orthogonal polynomials, which are outside the scope of this paper since the Hahn's property they satisfy can not be written in terms of the forward difference operator $\Delta$.

Next we introduce the concept of semiclassical discrete linear functional [see Ref. 17].

DEFINITION 2.10 A linear functional $u$ is said to be a semiclassical discrete linear functional if $u$ is weakly quasi-definite and there exist two polynomials $\phi$ and $\psi$ such that

$$
\Delta[\phi(x) u]=\psi(x) u
$$

where $\operatorname{deg}(\phi)=t \geq 0$ and $\operatorname{deg}(\psi)=p \geq 1$. A MOPS with respect to a semiclassical discrete functional $u$ is called a semiclassical discrete MOPS.

It is possible to associate with (2.6) a nonnegative integer $s=\max \{\operatorname{deg}(\psi)-1$, $\operatorname{deg}(\phi)-2\}$, but a semiclassical discrete functional $u$ satisfies an infinite number of equations as (2.6). It is enough to multiply both sides of (2.6) by a polynomial $f$ with $\operatorname{deg}(f)=q$ and from Proposition 2.7 we have $\Delta[f(x+1) \phi(x) u]=(\phi(x) \Delta f(x)+f(x) \psi(x)) u$. So $u$ fulfills also $\Delta\left[\phi_{1}(x) u\right]=\psi_{1}(x) u$, where $\phi_{1}(x)=f(x+1) \phi(x)$ and $\psi_{1}(x)=\phi(x) \Delta f(x)+f(x) \psi(x)$. From (2.6) we have $s_{1}=\max \left\{p_{1}-1, t_{1}-2\right\} \leq s+q$. Hence, we can associate with a semiclassical discrete functional $u$ a set of nonnegative integer numbers $h(u)$.

DEFINITION 2.11 Let $u$ be a semiclassical discrete functional. The minimum of the set $h(u)$ is called the class of $u$. When $s$ is the class of $u$, then the sequence of polynomials orthogonal with respect to $u$ is said to be of class $s$.

Note that classical discrete polynomials are semiclassical of class 0 .

DEFINITION 2.12 Let $\left\{\mathrm{P}_{n}(x)\right\}_{n=0}^{M}$ be the MOPS associated with the weakly quasi-definite linear functional $u$ of order $M$. The family of linear functionals $\left\{\alpha_{n}\right\}_{n}$ defined by $\left\langle\alpha_{n}, \mathrm{P}_{m}\right\rangle=\delta_{n m}, 0 \leq n, m \leq M$, is called the dual basis of $\left\{\mathrm{P}_{n}(x)\right\}_{n=0}^{M}$. 
In fact,

$$
\alpha_{n}=\frac{\mathrm{P}_{n}(x)}{\left\langle u, \mathrm{P}_{n}^{2}(x)\right\rangle} u, \quad 0 \leq n \leq M .
$$

An immediate consequence of the above equation can be stated as follows.

PROPOSITION 2.13 Let $\left\{\mathrm{P}_{n}(x)\right\}_{n=0}^{M}$ be the MOPS associated with the weakly quasi-definite linear functional $u$ of order $M \geq 1$ and let $\left\{\mathrm{Q}_{n}(x)\right\}_{n=0}^{M}{ }^{1}$ as in (2.3). If we denote by $\left\{\alpha_{n}\right\}_{n=0}^{M}$ and $\left\{\breve{\alpha}_{n}\right\}_{n=0}^{M}{ }^{1}$ the corresponding dual bases, then $\Delta \breve{\alpha}_{n}=-(n+1) \alpha_{n+1}, 0 \leq n \leq M-1$.

\section{D-COHERENT PAIRS}

DEFINITION 3.1 Let $u_{0}$ and $u_{1}$ be two weakly quasi-definite linear functionals of order $M_{0}$ and $M_{1}$, whose MOPS are $\left\{\mathrm{P}_{n}(x)\right\}_{n=0}^{M_{0}}$ and $\left\{\mathrm{T}_{n}(x)\right\}_{n=0}^{M_{1}}$ respectively, with $M_{0} \geq 2$ and $M_{1} \geq 1$. The pair $\left(u_{0}, u_{1}\right)$ is called $a \Delta$-coherent pair of linear functionals if

$$
\mathrm{T}_{n}(x)=\frac{\Delta \mathrm{P}_{n+1}(x)}{n+1}-\sigma_{n} \frac{\Delta \mathrm{P}_{n}(x)}{n}, \quad 1 \leq n \leq \min \left\{M_{0}-1, M_{1}\right\}
$$

where $\left\{\sigma_{n}\right\}_{n}$ is a sequence of non-zero complex numbers.

Example 3.2 Let $\mathbb{N}$ be the set of positive integers. Monic $\operatorname{Kravchuk}$ polynomials $\mathrm{k}_{n}^{(p)}(x ; N)$ have the following representation in terms of hypergeometric series [Ref. 21, p. 51]

$$
\begin{gathered}
\mathrm{k}_{n}^{(p)}(x ; N)=(-p)^{n}(N-n+1)_{n}{ }_{2} \mathrm{~F}_{1}\left(\begin{array}{c|c}
-n,-x & \frac{1}{p} \\
-N &
\end{array}\right), \\
0<p<1, \quad N \in \mathbb{N}, \quad 0 \leq n \leq N, \quad N \geq 2,
\end{gathered}
$$

where $(a)_{n}$ denotes the Pochhammer symbol [2], which is defined by

$$
(a)_{0}=1, \quad(a)_{n}=a(a+1) \cdots(a+n-1), \quad n \geq 1 .
$$

Since Kravchuk polynomials satisfy [see, for example, Ref. 10]

$$
\mathrm{k}_{n}^{(p)}(x ; N)=\frac{\Delta \mathrm{k}_{n+1}^{(p)}(x ; N)}{n+1}-\sigma_{n} \frac{\Delta \mathrm{k}_{n}^{(p)}(x ; N)}{n}, \quad \text { with } \sigma_{n}=n p, \quad 1 \leq n \leq N-1,
$$

if we denote by $u^{(p, N)}$ the Kravchuk linear functional of order $N$ (the binomial distribution from probability theory) given by

$$
\left\langle u^{(p, N)}, r\right\rangle=\sum_{s=0}^{N}\left(\begin{array}{c}
N \\
s
\end{array}\right) p^{s}(1-p)^{N} s \quad r(s), \quad 0<p<1, \quad N \in \mathbb{N}, \quad \text { for every } r \in \mathbb{P},
$$

we have that $\left(u^{(p, N)}, u^{(p, N)}\right)$ is a $\Delta$-coherent pair of linear functionals, if $N \geq 2$. 
PROPOSITION 3.3 Let $\left(u_{0}, u_{1}\right)$ be a $\Delta$-coherent pair of linear functionals and let $\left\{\alpha_{n}^{(0)}\right\}_{n=0}^{M_{0}}$ and $\left\{\alpha_{n}^{(1)}\right\}_{n=0}^{M_{1}}$ be the dual bases of $u_{0}$ and $u_{1}$, respectively, with $M_{0} \geq 2$ and $M_{1} \geq 1$. If we denote by $\left\{\breve{\alpha}_{n}^{(0)}\right\}_{n=0}^{M_{0}}{ }^{1}$ the dual basis corresponding to $\left\{\mathrm{Q}_{n}(x)\right\}_{n=0}^{M_{0}}{ }^{1}$ defined in (2.3), then we have

$$
\begin{gathered}
\breve{\alpha}_{n}^{(0)}=\alpha_{n}^{(1)}-\sigma_{n+1} \alpha_{n+1}^{(1)}, \quad 0 \leq n \leq \min \left\{M_{0}-2, M_{1}-1\right\}, \\
(n+1) \alpha_{n+1}^{(0)}=\sigma_{n+1} \Delta \alpha_{n+1}^{(1)}-\Delta \alpha_{n}^{(1)}, \quad 0 \leq n \leq \min \left\{M_{0}-2, M_{1}-1\right\} .
\end{gathered}
$$

Proof Let $\mathbb{P}_{M_{1}}$ be the space of polynomials of degree at most $M_{1}$ and let $\mathbb{P}_{M}^{\prime}$ be its algebraic dual space. Equation (3.5) is a consequence of (3.1) and using that $\left\{\alpha_{n}^{(1)}\right\}_{n=0}^{M_{1}}$ is a basis of $\mathbb{P}_{M_{1}}^{\prime}$. Applying the $\Delta$ operator to (3.5) and using Proposition 2.13, (3.6) is obtained.

THEOREM 3.4 Let $\left(u_{0}, u_{1}\right)$ be a $\Delta$-coherent pair of linear functionals and let $\left\{\mathrm{P}_{n}(x)\right\}_{n=0}^{M_{0}}$ and $\left\{\mathrm{T}_{n}(x)\right\}_{n=0}^{M_{1}}$ be the corresponding MOPS associated with $u_{0}$ and $u_{1}$, respectively, with $M_{0} \geq 2$ and $M_{1} \geq 1$. Then

(i) The functional $u_{1}$ is a semiclassical discrete linear functional of class at most 1 . That is, there exist two polynomials $\phi_{1}$ and $\psi_{1}$, of degree at most 3 and 2 , respectively, such that

$$
\Delta\left[\phi_{1}(x) u_{1}\right]=\psi_{1}(x) u_{1}
$$

Their explicit expressions are

$$
\begin{aligned}
& \phi_{1}(x)=2 \frac{\mathrm{P}_{2}(x+1)}{\left\langle u_{0}, \mathrm{P}_{2}^{2}\right\rangle} \mathrm{c}_{1}(x)-\frac{\mathrm{P}_{1}(x+1)}{\left\langle u_{0}, \mathrm{P}_{1}^{2}\right\rangle} \mathrm{c}_{2}(x), \\
& \psi_{1}(x)=\frac{\mathrm{P}_{1}(x)}{\left\langle u_{0}, \mathrm{P}_{1}^{2}\right\rangle} \Delta \mathrm{c}_{2}(x-1)-2 \frac{\mathrm{P}_{2}(x)}{\left\langle u_{0}, \mathrm{P}_{2}^{2}\right\rangle} \Delta \mathrm{c}_{1}(x-1)+\Delta \phi_{1}(x-1),
\end{aligned}
$$

where

$$
\mathrm{c}_{n+1}(x):=\sigma_{n+1} \frac{\mathrm{T}_{n+1}(x)}{\left\langle u_{1}, \mathrm{~T}_{n+1}^{2}\right\rangle}-\frac{\mathrm{T}_{n}(x)}{\left\langle u_{1}, \mathrm{~T}_{n}^{2}\right\rangle}, \quad 0 \leq n \leq \min \left\{M_{0}-2, M_{1}-1\right\} .
$$

(ii) There exist polynomials $\mathrm{A}_{3}$ and $\mathrm{B}_{2}$ of degree at most 3 and 2 , respectively, such that

$$
\mathrm{A}_{3}(x) u_{0}=\mathrm{B}_{2}(x) u_{1}
$$

where

$\mathrm{A}_{3}(x)=\phi_{1}(x-1), \quad \mathrm{B}_{2}(x)=\mathrm{c}_{1}(x-1) \Delta \mathrm{c}_{2}(x-1)-\mathrm{c}_{2}(x-1) \Delta \mathrm{c}_{1}(x-1)$.

(iii) The functional $u_{0}$ is a semiclassical discrete linear functional of class at most 6 since it verifies the distributional equation $\Delta\left[\phi_{0}(x) u_{0}\right]=\psi_{0}(x) u_{0}$, where $\phi_{0}$ and $\psi_{0}$ are the polynomials of degree at most 8 and 7 given by

$$
\begin{gathered}
\phi_{0}(x)=\phi_{1}(x) \phi_{1}(x-1) \mathrm{B}_{2}(x), \\
\psi_{0}(x)=\left\{\mathrm{B}_{2}(x-1) \psi_{1}(x)+\phi_{1}(x) \Delta \mathrm{B}_{2}(x-1)\right\} \mathrm{A}_{3}(x-1)+\phi_{1}(x) \mathrm{A}_{3}(x) \Delta \mathrm{B}_{2}(x) .
\end{gathered}
$$


Proof Let us write (3.6) using (2.7)

$$
(n+1) \frac{\mathrm{P}_{n+1}(x)}{\left\langle u_{0}, \mathrm{P}_{n+1}^{2}\right\rangle} u_{0}=\Delta\left[\mathrm{c}_{n+1}(x) u_{1}\right], \quad 0 \leq n \leq \min \left\{M_{0}-2, M_{1}-1\right\} .
$$

For $n=0$ and $n=1$ we get in (3.15)

$$
\begin{aligned}
\frac{\mathrm{P}_{1}(x)}{\left\langle u_{0}, \mathrm{P}_{1}^{2}\right\rangle} u_{0} & =\Delta\left[\mathrm{c}_{1}(x) u_{1}\right]=\mathrm{c}_{1}(x-1) \Delta u_{1}+\Delta \mathrm{c}_{1}(x-1) u_{1}, \\
2 \frac{\mathrm{P}_{2}(x)}{\left\langle u_{0}, \mathrm{P}_{2}^{2}\right\rangle} u_{0} & =\Delta\left[\mathrm{c}_{2}(x) u_{1}\right]=\mathrm{c}_{2}(x-1) \Delta u_{1}+\Delta \mathrm{c}_{2}(x-1) u_{1} .
\end{aligned}
$$

(i) From (3.16) it follows that

$$
\begin{aligned}
& \left(2 \frac{\mathrm{P}_{2}(x)}{\left\langle u_{0}, \mathrm{P}_{2}^{2}\right\rangle} \mathrm{c}_{1}(x-1)-\frac{\mathrm{P}_{1}(x)}{\left\langle u_{0}, \mathrm{P}_{1}^{2}\right\rangle} \mathrm{c}_{2}(x-1)\right) \Delta u_{1} \\
& \quad+\left(2 \frac{\mathrm{P}_{2}(x)}{\left\langle u_{0}, \mathrm{P}_{2}^{2}\right\rangle} \Delta \mathrm{c}_{1}(x-1)-\frac{\mathrm{P}_{1}(x)}{\left\langle u_{0}, \mathrm{P}_{1}^{2}\right\rangle} \Delta \mathrm{c}_{2}(x-1)\right) u_{1}=0 .
\end{aligned}
$$

On the other hand,

$$
\begin{aligned}
& \Delta\left[\left(2 \frac{\mathrm{P}_{2}(x+1)}{\left\langle u_{0}, \mathrm{P}_{2}^{2}\right\rangle} \mathrm{c}_{1}(x)-\frac{\mathrm{P}_{1}(x+1)}{\left\langle u_{0}, \mathrm{P}_{1}^{2}\right\rangle} \mathrm{c}_{2}(x)\right) u_{1}\right] \\
& \quad=\left(\frac{\mathrm{P}_{1}(x)}{\left\langle u_{0}, \mathrm{P}_{1}^{2}\right\rangle} \Delta \mathrm{c}_{2}(x-1)-2 \frac{\mathrm{P}_{2}(x)}{\left\langle u_{0}, \mathrm{P}_{2}^{2}\right\rangle} \Delta \mathrm{c}_{1}(x-1)+\Delta \phi_{1}(x-1)\right) u_{1} .
\end{aligned}
$$

Hence, $\Delta\left[\phi_{1}(x) u_{1}\right]=\psi_{1}(x) u_{1}$, where $\phi_{1}$ and $\psi_{1}$ are given in (3.8) and (3.9), respectively. Thus (i) follows.

(ii) Eliminating $\Delta u_{1}$ in the system (3.16) we obtain $\mathrm{A}_{3}(x) u_{0}=\mathrm{B}_{2}(x) u_{1}$, where polynomials $\mathrm{A}_{3}$ and $\mathrm{B}_{2}$ are given in (3.12).

(iii) Finally, by using Proposition 2.7 appropriately we have

$$
\begin{aligned}
& \Delta\left[\phi_{1}(x) \phi_{1}(x-1) \mathrm{B}_{2}(x) u_{0}\right] \\
& \quad=\Delta\left[\phi_{1}(x) \mathrm{B}_{2}(x) \phi_{1}(x-1) u_{0}\right]=\Delta\left[\phi_{1}(x) \mathrm{B}_{2}^{2}(x) u_{1}\right] \\
& \quad=\mathrm{B}_{2}^{2}(x-1) \Delta\left[\phi_{1}(x) u_{1}\right]+\Delta \mathrm{B}_{2}^{2}(x-1) \phi_{1}(x) u_{1} \\
& \quad=\mathrm{B}_{2}^{2}(x-1) \psi_{1}(x) u_{1}+\left(\mathrm{B}_{2}(x-1) \Delta \mathrm{B}_{2}(x-1)+\mathrm{B}_{2}(x) \Delta \mathrm{B}_{2}(x-1)\right) \phi_{1}(x) u_{1} \\
& \quad=\phi_{1}(x) \Delta \mathrm{B}_{2}(x-1) \mathrm{A}_{3}(x) u_{0}+\left\{\mathrm{B}_{2}(x-1) \psi_{1}(x)+\phi_{1}(x) \Delta \mathrm{B}_{2}(x-1)\right\} \mathrm{A}_{3}(x-1) u_{0} .
\end{aligned}
$$

\section{GENERAL PROBLEM OF $\triangle$-COHERENCE}

In Theorem 3.4 we have proved that if $\left(u_{0}, u_{1}\right)$ is a $\Delta$-coherent pair of linear functionals, then both $u_{0}, u_{1}$ are semiclassical discrete functionals of class at most 6 and 1, respectively. The main goal of this section is to prove that if $\left(u_{0}, u_{1}\right)$ is a $\Delta$-coherent pair of linear functionals then at least one of the functionals $u_{0}, u_{1}$ must be a classical discrete functional under certain conditions on the order of $u_{0}$ and $u_{1}$. The proof of this statement will consist in 3 steps. Let us denote by $\xi$ and $\eta$ the zeros of the polynomial $\mathrm{B}_{2}(x)$ defined in (3.12). In the first one, we prove that if $\eta=\xi+1, u_{0}$ must be a classical discrete functional (Theorem 4.2). In the 
second step we prove that if $\eta \neq \xi$ and $\eta \neq \xi+1$ then $u_{1}$ must be a classical discrete functional (Theorem 4.6). Finally, as a remark, we prove that the case $\eta=\xi$ can not be hold.

PROPOSITION 4.1 Let $\left(u_{0}, u_{1}\right)$ be a $\Delta$-coherent pair of linear functionals, and let $\left\{\mathrm{P}_{n}(x)\right\}_{n=0}^{M_{0}}$ and $\left\{\mathrm{T}_{n}(x)\right\}_{n=0}^{M_{1}}$ the corresponding MOPS associated with $u_{0}$ and $u_{1}$, respectively with $M_{1}>3$. Let $\mathrm{c}_{n}(x)$ be the polynomials defined in (3.10). For each $1 \leq n \leq \min \left\{M_{0}-1, M_{1}\right\}$, we have

$$
n \frac{\mathrm{P}_{n}(x)}{\left\langle u_{0}, \mathrm{P}_{n}^{2}\right\rangle} \mathrm{B}_{2}(x)=\mathrm{A}_{3}(x) \Delta \mathrm{c}_{n}(x-1)+\mathrm{c}_{n}(x-1) \pi(x),
$$

where the polynomials $\mathrm{A}_{3}(x)$ and $\mathrm{B}_{2}(x)$ are defined in (3.12) and $\pi(x):=\psi_{1}(x)-\Delta \mathrm{A}_{3}(x)$, with $\psi_{1}(x)$ given in (3.9).

Proof Using (3.11), (3.15) and Proposition 2.7 we obtain

$$
\begin{aligned}
n \frac{\mathrm{P}_{n}(x)}{\left\langle u_{0}, \mathrm{P}_{n}^{2}\right\rangle} \mathrm{B}_{2}(x) u_{1} & =n \frac{\mathrm{P}_{n}(x)}{\left\langle u_{0}, \mathrm{P}_{n}^{2}\right\rangle} \mathrm{A}_{3}(x) u_{0}=\mathrm{A}_{3}(x) \Delta\left[\mathrm{c}_{n}(x) u_{1}\right] \\
& =\mathrm{A}_{3}(x)\left\{\mathrm{c}_{n}(x-1) \Delta u_{1}+\Delta \mathrm{c}_{n}(x-1) u_{1}\right\}, \quad 1 \leq n \leq \min \left\{M_{0}-1, M_{1}\right\}
\end{aligned}
$$

From Proposition 2.7 and (3.7) we get

$$
\phi_{1}(x-1) \Delta u_{1}=\pi(x) u_{1}
$$

and then (4.1) holds, since $M_{1}>3$.

THEOREM 4.2 Let $\left(u_{0}, u_{1}\right)$ be a $\Delta$-coherent pair of linear functionals. Let $\xi$ and $\eta$ be the zeros of the polynomial $\mathrm{B}_{2}(x)$ defined in (3.12) and suppose that $\eta=\xi+1$, i.e., let

$$
\mathrm{B}_{2}(x)=\frac{\sigma_{1} \sigma_{2}}{\left\langle u_{1}, \mathrm{~T}_{1}^{2}\right\rangle\left\langle u_{1}, \mathrm{~T}_{2}^{2}\right\rangle}(x-\xi)(x-(\xi+1)) .
$$

Then,

(i) If the order of the linear functional $u_{1}$ is greater than 3, then the functional $\tilde{u}=(x-\xi) u_{1}$ is a classical discrete linear functional verifying

$$
\Delta[\tilde{\phi}(x) \tilde{u}]=\tilde{\psi}(x) \tilde{u}
$$

for some polynomials $\tilde{\phi}$ and $\tilde{\psi}$, with $\operatorname{deg}(\tilde{\phi}) \leq 2$ and $\operatorname{deg}(\tilde{\psi})=1$. Moreover,

$$
\tilde{\phi}(x-1) u_{0}=\frac{\sigma_{1} \sigma_{2}}{\left\langle u_{1}, \mathrm{~T}_{1}^{2}\right\rangle\left\langle u_{1}, \mathrm{~T}_{2}^{2}\right\rangle} \tilde{u} .
$$

(ii) The functional $u_{0}$ is a classical discrete linear functional satisfying

$$
\Delta\left[\tilde{\phi}(x-1) u_{0}\right]=\tilde{\psi}_{0}(x) u_{0}
$$

for some polynomial $\tilde{\psi}_{0}$ with $\operatorname{deg}\left(\tilde{\psi}_{0}\right)=1$. 
Proof We have proved in Theorem 3.4 that $u_{1}$ is a semiclassical discrete linear functional satisfying the distributional Eq. (3.7).

(i) From the definition of $\mathrm{B}_{2}(x)$ in (4.3) it follows

$$
\Delta \mathrm{B}_{2}(x)=2 \frac{\sigma_{1} \sigma_{2}}{\left\langle u_{1}, \mathrm{~T}_{1}^{2}\right\rangle\left\langle u_{1}, \mathrm{~T}_{2}^{2}\right\rangle}(x-\xi)
$$

Moreover, from (3.12) we get

$$
\Delta \mathrm{B}_{2}(x)=2 \frac{\sigma_{2}}{\left\langle u_{1}, \mathrm{~T}_{2}^{2}\right\rangle} \mathrm{c}_{1}(x)
$$

using (3.10). Thus we obtain that $\mathrm{c}_{1}(\xi)=0$. From (3.12) with $x=\xi+1$ it follows that

$$
0=\mathrm{B}_{2}(\xi+1)=\mathrm{c}_{1}(\xi) \Delta \mathrm{c}_{2}(\xi)-\mathrm{c}_{2}(\xi) \Delta \mathrm{c}_{1}(\xi)=-\mathrm{c}_{2}(\xi) \frac{\sigma_{1}}{\left\langle u_{1}, \mathrm{~T}_{1}^{2}\right\rangle}
$$

Thus $c_{2}(\xi)=0$. Since $c_{1}(\xi)=c_{2}(\xi)=0$ from (3.8) we obtain $\phi_{1}(\xi)=0$ as well as $\psi_{1}(\xi)=0$ using (3.8) and (3.9).

Hence, we can write

$$
\phi_{1}(x)=(x-\xi) \tilde{\phi}(x), \quad \psi_{1}(x)=(x-\xi) \tilde{\psi}(x)
$$

Let us define $\tilde{u}=(x-\xi) u_{1}$. From (3.7) and the definition of polynomials $\tilde{\phi}$ and $\tilde{\psi}$, it follows that $\tilde{u}$ satisfies (4.4). In Theorem 3.4 we have proved that $\operatorname{deg}\left(\phi_{1}\right)$ is at most 3, so $\operatorname{deg}(\tilde{\phi}) \leq 2$. Since $\operatorname{deg}\left(\psi_{1}\right)$ is at most 2 , we deduce $\operatorname{deg}(\tilde{\psi}) \leq 1$. If we prove that $\tilde{\psi}$ can not be a constant polynomial then we deduce part (i) of the Theorem. Indeed, we shall distinguish two situations:

(1) If $\tilde{\psi}$ is a non-zero constant $v$, then $\left\langle u_{1}, v(x-\xi)\right\rangle=\left\langle v(x-\xi) u_{1}, 1\right\rangle=\langle v \tilde{u}, 1\rangle=$ $\langle\Delta[\tilde{\phi}(x) \tilde{u}], 1\rangle=0$. Hence $\mathrm{T}_{1}(x)=x-\xi$. From (3.10) then $\mathrm{c}_{1}(\xi) \neq 0$ and this contradicts that $\mathrm{c}_{1}(\xi)=0$.

(2) Suppose that $\tilde{\psi} \equiv 0$ and let us denote by $x^{[n]}=x(x-1) \cdots(x-n+1), x^{[0]}=1$. Since $\Delta x^{[n+1]}=x^{[n]}(n+1)$ for each $n \geq 0$, it should be

$$
\left\langle\phi_{1}(x) u_{1}, x^{[n]}\right\rangle=-\left\langle\Delta\left[\phi_{1}(x) u_{1}\right], \frac{x^{[n+1]}}{n+1}\right\rangle=-\left\langle\Delta[\tilde{\phi}(x) \tilde{u}], \frac{x^{[n+1]}}{n+1}\right\rangle=\left\langle\tilde{\psi}(x) \tilde{u}, x^{[n]}\right\rangle=0 .
$$

So, $\left\langle\phi_{1}(x) u_{1}, p(x)\right\rangle=0$ for every $p \in \mathbb{P}$, and then $u_{1}$ should not be a weakly quasidefinite linear functional of order greater than 3 . Hence $\tilde{\psi} \not \equiv 0$.

From the above situations we conclude that $\operatorname{deg}(\tilde{\psi})=1$.

Moreover, since $\mathrm{c}_{1}(\xi)=\mathrm{c}_{2}(\xi)=0$ then $\mathrm{c}_{1}(x)$ divides $\mathrm{c}_{2}(x)$. From (3.11) and (3.12) we can write

$$
(x-(\xi+1)) \tilde{\phi}(x-1) u_{0}=\mathrm{B}_{2}(x) u_{1} .
$$


Multiplying both sides of this equality by $(x-(\xi+1))^{1}$, and using $(2.1)$ we obtain

$$
\tilde{\phi}(x-1) u_{0}=\frac{\sigma_{1} \sigma_{2}}{\left\langle u_{1}, \mathrm{~T}_{1}^{2}\right\rangle\left\langle u_{1}, \mathrm{~T}_{2}^{2}\right\rangle}(x-\xi) u_{1}+\delta_{\xi+1}\left[\left(\tilde{\phi}(x-1) u_{0}\right)_{0}-\left((x-\xi) u_{1}\right)_{0}\right] .
$$

With an appropriate choice of the first moments of the functionals $u_{0}$ and $u_{1}$, it yields

$$
\tilde{\phi}(x-1) u_{0}=\frac{\sigma_{1} \sigma_{2}}{\left\langle u_{1}, \mathrm{~T}_{1}^{2}\right\rangle\left\langle u_{1}, \mathrm{~T}_{2}^{2}\right\rangle}(x-\xi) u_{1}
$$

Thus we get (4.5).

(ii) From the above equality and (3.15)

$$
\Delta\left[\tilde{\phi}(x-1) u_{0}\right]=\Delta\left[\frac{\sigma_{1} \sigma_{2}}{\left\langle u_{1}, \mathrm{~T}_{1}^{2}\right\rangle\left\langle u_{1}, \mathrm{~T}_{2}^{2}\right\rangle}(x-\xi) u_{1}\right]=\frac{\sigma_{2}}{\left\langle u_{1}, \mathrm{~T}_{2}^{2}\right\rangle} \Delta\left[\mathrm{c}_{1}(x) u_{1}\right]=\frac{\sigma_{2}}{\left\langle u_{1}, \mathrm{~T}_{2}^{2}\right\rangle} \frac{\mathrm{P}_{1}(x)}{\left\langle u_{0}, \mathrm{P}_{1}^{2}\right\rangle} u_{0} .
$$

If we define $\tilde{\psi}_{0}(x)=\left(\sigma_{2} /\left\langle u_{1}, \mathrm{~T}_{2}^{2}\right\rangle\right)\left(\mathrm{P}_{1}(x) /\left\langle u_{0}, \mathrm{P}_{1}^{2}\right\rangle\right)$, then $\operatorname{deg}\left(\tilde{\psi}_{0}\right)=1$ and (4.6) holds.

For the remaining steps described in the introduction of this section, some previous lemmas are needed.

LEMMA 4.3 Let $\left(u_{0}, u_{1}\right)$ be a $\Delta$-coherent pair of linear functionals and $\mathrm{A}_{3}(x)$ and $\mathrm{B}_{2}(x)$ the polynomials defined in (3.12). Let $\xi$ be a zero of $\mathrm{B}_{2}(x)$ such that $\mathrm{A}_{3}(\xi) \neq 0$. Then, there exists a non-zero parameter $k$ independent of $n$, such that

$$
\mathrm{c}_{n}(\xi-1)+k \Delta \mathrm{c}_{n}(\xi-1)=0, \quad 1 \leq n \leq \min \left\{M_{0}-1, M_{1}\right\},
$$

where $M_{0} \geq 2$ and $M_{1} \geq 1$ are the order of $u_{0}$ and $u_{1}$, respectively.

Proof Since $\mathrm{B}_{2}(\xi)=0$, (4.1) for $n=1$ and $x=\xi$ reads as

$$
0=\mathrm{A}_{3}(\xi) \Delta \mathrm{c}_{1}(\xi-1)+\mathrm{c}_{1}(\xi-1) \pi(\xi) .
$$

Since $\Delta \mathrm{c}_{1}(\xi-1)=\sigma_{1} /\left\langle u_{1}, \mathrm{~T}_{1}^{2}\right\rangle$ is a non-zero constant then $\pi(\xi) \neq 0$ and

$$
\mathrm{c}_{1}(\xi-1)=-\frac{\mathrm{A}_{3}(\xi) \sigma_{1}}{\pi(\xi)\left\langle u_{1}, \mathrm{~T}_{1}^{2}\right\rangle} \neq 0 .
$$

If we define

$$
k=\frac{\mathrm{A}_{3}(\xi)}{\pi(\xi)}=-\frac{\mathrm{c}_{1}(\xi-1)}{\Delta \mathrm{c}_{1}(\xi-1)}
$$

our result follows from (4.1) for $1 \leq n \leq \min \left\{M_{0}-1, M_{1}\right\}$.

LEMMA 4.4 Let $\left(u_{0}, u_{1}\right)$ be a $\Delta$-coherent pair of linear functionals. Suppose that there exist parameters $\xi_{1}, \xi_{2}, k_{1} \neq 0$ and $k_{2} \neq 0$ such that

$$
\mathrm{c}_{n}\left(\xi_{1}\right)+k_{1} \Delta \mathrm{c}_{n}\left(\xi_{1}\right)=0, \quad \text { and } \mathrm{c}_{n}\left(\xi_{2}\right)+k_{2} \Delta \mathrm{c}_{n}\left(\xi_{2}\right)=0, \quad 1 \leq n \leq \min \left\{M_{0}-1, M_{1}\right\},
$$


where $M_{0}$ and $M_{1}$ are the order of $u_{0}$ and $u_{1}$, respectively. If $M_{1} \geq 2, M_{1} \geq 3$, and $\left|\xi_{1}-\xi_{2}\right| \neq 1$ then $\xi_{1}=\xi_{2}$ and $k_{1}=k_{2}$.

Proof From (3.10), Eqs. (4.7) can be written

$$
\begin{aligned}
& \sigma_{n}\left\{\frac{\mathrm{T}_{n}\left(\xi_{j}\right)}{\left\langle u_{1}, \mathrm{~T}_{n}^{2}\right\rangle}+k_{j} \frac{\Delta \mathrm{T}_{n}\left(\xi_{j}\right)}{\left\langle u_{1}, \mathrm{~T}_{n}^{2}\right\rangle}\right\} \\
& \quad=\frac{\mathrm{T}_{n} 1\left(\xi_{j}\right)}{\left\langle u_{1}, \mathrm{~T}_{n}^{2}{ }_{1}\right\rangle}+k_{j} \frac{\Delta \mathrm{T}_{n} 1\left(\xi_{j}\right)}{\left\langle u_{1}, \mathrm{~T}_{n}^{2}{ }_{1}\right\rangle}, \quad j=1,2, \quad 1 \leq n \leq \min \left\{M_{0}-1, M_{1}\right\} .
\end{aligned}
$$

Let us denote

$$
\mathrm{h}_{n}^{(j)}\left(\xi_{j}\right)=\frac{\mathrm{T}_{n}\left(\xi_{j}\right)}{\left\langle u_{1}, \mathrm{~T}_{n}^{2}\right\rangle}+k_{j} \frac{\Delta \mathrm{T}_{n}\left(\xi_{j}\right)}{\left\langle u_{1}, \mathrm{~T}_{n}^{2}\right\rangle}, \quad j=1,2, \quad 1 \leq n \leq \min \left\{M_{0}-1, M_{1}\right\}
$$

and observe that $\mathrm{h}_{0}^{(j)}\left(\xi_{j}\right)=1 /\left\langle u_{1}, 1\right\rangle$. For each $1 \leq n \leq \min \left\{M_{0}-1, M_{1}\right\}$ and for $j=1,2$ we write $\sigma_{n} \mathrm{~h}_{n}^{(j)}\left(\xi_{j}\right)=\mathrm{h}_{n}^{(j)}{ }_{1}\left(\xi_{j}\right)$, so $\mathrm{h}_{n}^{(j)}\left(\xi_{j}\right) \neq 0$, for every $1 \leq n \leq \min \left\{M_{0}-1, M_{1}\right\}$, and we get

$$
\frac{\mathrm{h}_{n}^{(1)}\left(\xi_{1}\right)}{\mathrm{h}_{n}^{(2)}\left(\xi_{2}\right)}=\frac{\mathrm{h}_{n}^{(1)}\left(\xi_{1}\right)}{\mathrm{h}_{n}^{(2)}\left(\xi_{2}\right)}, \quad 1 \leq n \leq \min \left\{M_{0}-1, M_{1}\right\}
$$

Repeating this process

$$
\frac{\mathrm{h}_{n}^{(1)}\left(\xi_{1}\right)}{\mathrm{h}_{n}^{(2)}\left(\xi_{2}\right)}=\frac{\mathrm{h}_{0}^{(1)}\left(\xi_{1}\right)}{\mathrm{h}_{0}^{(2)}\left(\xi_{2}\right)}=\frac{1 /\left\langle u_{1}, 1\right\rangle}{1 /\left\langle u_{1}, 1\right\rangle}=1, \quad 1 \leq n \leq \min \left\{M_{0}-1, M_{1}\right\},
$$

i.e., $\mathrm{h}_{n}^{(1)}\left(\xi_{1}\right)=\mathrm{h}_{n}^{(2)}\left(\xi_{2}\right)$ for $1 \leq n \leq \min \left\{M_{0}-1, M_{1}\right\}$ or, equivalently,

$$
\mathrm{T}_{n}\left(\xi_{1}\right)+k_{1} \Delta \mathrm{T}_{n}\left(\xi_{1}\right)=\mathrm{T}_{n}\left(\xi_{2}\right)+k_{2} \Delta \mathrm{T}_{n}\left(\xi_{2}\right), \quad 1 \leq n \leq \min \left\{M_{0}-1, M_{1}\right\}
$$

So from the initial problem of characterizing $\xi_{1}, \xi_{2}, k_{1}$ and $k_{2}$ such that (4.7) holds, we propose a new problem: determine $\xi_{1}, \xi_{2}, k_{1}$ and $k_{2}$ such that (4.8) holds.

In order to solve it, we study a more general one: Find all $\mu, v, \delta$ and $\eta$ such that

$$
\mu \mathrm{T}_{n}\left(\xi_{1}\right)+v \Delta \mathrm{T}_{n}\left(\xi_{1}\right)=\delta \mathrm{T}_{n}\left(\xi_{2}\right)+\eta \Delta \mathrm{T}_{n}\left(\xi_{2}\right), \quad 1 \leq n \leq \min \left\{M_{0}-1, M_{1}\right\}
$$

Since $\left\{\mathrm{T}_{n}(x)\right\}_{n=0}^{M_{1}}$ is a MOPS, it satisfies a three-term recurrence relation [6] which can be written $\mathrm{T}_{n+1}(x)=\left(x-\beta_{n}^{\mathrm{T}}\right) \mathrm{T}_{n}(x)-\gamma_{n}^{\mathrm{T}} \mathrm{T}_{n} 1(x), 0 \leq n \leq M_{1}-1$ with the initial conditions $\mathrm{T}_{1}(x)=0$ and $\mathrm{T}_{0}(x)=1$. If we apply the $\Delta$ operator to this relation

$$
\Delta \mathrm{T}_{n+1}(x)=\mathrm{T}_{n}(x)+\left(x-\beta_{n}^{\mathrm{T}}+1\right) \Delta \mathrm{T}_{n}(x)-\gamma_{n}^{\mathrm{T}} \Delta \mathrm{T}_{n} \quad 1(x), \quad 0 \leq n \leq M_{1}-1 .
$$

Using the three-term recurrence relation for $\left\{\mathrm{T}_{n}(x)\right\}_{n}$ and (4.10) a new equation is obtained:

$$
\begin{gathered}
\left(v+\mu \xi_{1}\right) \mathrm{T}_{n}\left(\xi_{1}\right)+v\left(1+\xi_{1}\right) \Delta \mathrm{T}_{n}\left(\xi_{1}\right)=\left(\eta+\delta \xi_{2}\right) \mathrm{T}_{n}\left(\xi_{2}\right)+\eta\left(1+\xi_{2}\right) \Delta \mathrm{T}_{n}\left(\xi_{2}\right), \\
0 \leq n \leq M_{1} .
\end{gathered}
$$


Let us repeat the process from (4.9) to (4.11), but starting with (4.11) instead of (4.9). Finally, mimicking the process starting with this new last equation we find an homogeneous system of four linear equations with variables $\mathrm{T}_{n}\left(\xi_{1}\right), \mathrm{T}_{n}\left(\xi_{2}\right), \Delta \mathrm{T}_{n}\left(\xi_{1}\right)$ and $\Delta \mathrm{T}_{n}\left(\xi_{2}\right)$. The determinant of the matrix of coefficients is, after replacing $\mu=1, v=k_{1}, \delta=1$ and $\eta=k_{2}$,

$$
k_{1} k_{2}\left(k_{1}-1\right)\left(k_{2}-1\right)\left(\xi_{1}-\xi_{2}\right)^{2}\left(\xi_{2}-\xi_{1}-1\right)\left(\xi_{2}-\xi_{1}+1\right) .
$$

Then, we need to study how are the solutions of this linear system, depending on the value of (4.12).

If the determinant (4.12) is different of zero, then the solution of the linear system is

$$
\mathrm{T}_{n}\left(\xi_{i}\right)=\Delta \mathrm{T}_{n}\left(\xi_{i}\right)=0, \quad 3 \leq n \leq M_{1}, \quad i=1,2 .
$$

Hence it should be $\mathrm{T}_{n}\left(\xi_{1}\right)=\mathrm{T}_{n}\left(\xi_{2}\right)=\mathrm{T}_{n}\left(\xi_{1}+1\right)=\mathrm{T}_{n}\left(\xi_{2}+1\right)=0$, for every $3 \leq n \leq M_{1}$, but this contradicts that $\left\{\mathrm{T}_{n}(x)\right\}_{n=0}^{M_{1}}$ is a MOPS.

Now we discuss what happens when (4.12) vanishes.

(1) If $k_{i}=1(i=1,2)$ then the solution of the system is $\mathrm{T}_{n}\left(\xi_{i}\right)=-\Delta \mathrm{T}_{n}\left(\xi_{i}\right), \mathrm{T}_{n}\left(\xi_{j}\right)=$ $\Delta \mathrm{T}_{n}\left(\xi_{j}\right)=0$, for every $3 \leq n \leq M_{1}$. Hence $\mathrm{T}_{n}\left(\xi_{i}+1\right)=\mathrm{T}_{n}\left(\xi_{j}\right)=\mathrm{T}_{n}\left(\xi_{j}+1\right)=0$ for every $3 \leq n \leq M_{1}$. But since $\left\{\mathrm{T}_{n}(x)\right\}_{n=0}^{M_{1}}$ is a MOPS these equations can not be held.

(2) If $k_{i} \neq 1(i=1,2)$,

(a) Suppose $\xi_{1}=\xi_{2}$. Then, it is trivial to check that $k_{1}=k_{2}$.

(b) The case $\left|\xi_{1}-\xi_{2}\right|=1$ can not be held because of the hypothesis of the Lemma.

LEMMA 4.5 Let $\left(u_{0}, u_{1}\right)$ be a $\Delta$-coherent pair of linear functionals, where $M_{0} \geq 2$ and $M_{1} \geq 4$ are the order of $u_{0}$ and $u_{1}$, respectively. Let $\mathrm{A}_{3}(x)$ and $\mathrm{B}_{2}(x)$ be the polynomials defined in (3.12) and $\mathrm{c}_{n}(x)$ the polynomials defined in (3.10). If $\mathrm{B}_{2}(x)$ has not a double zero and that no zero of $\mathrm{B}_{2}(x)$ is a root of $\Delta \mathrm{B}_{2}(x)=0$, then there exists a parameter $\xi$ such that $\mathrm{B}_{2}(\xi)=\mathrm{A}_{3}(\xi)=0$. Furthermore, we have $\mathrm{c}_{1}(\xi) \neq 0, \mathrm{c}_{1}(\xi-1) \neq 0$ and $\pi(\xi)=0$.

Proof Let us denote $\xi_{1}$ and $\xi_{2}$ the zeros of $\mathrm{B}_{2}(x)$. If both $\xi_{i}(i=1,2)$ are not zeros of $\mathrm{A}_{3}(x)$, we can apply Lemma 4.3 to obtain two constants $k_{1} \neq 0$ and $k_{2} \neq 0$ such that $\mathrm{c}_{n}\left(\xi_{1}-1\right)+k_{1} \Delta \mathrm{c}_{n}\left(\xi_{1}-1\right)=0$ and $\mathrm{c}_{n}\left(\xi_{2}-1\right)+k_{2} \Delta \mathrm{c}_{n}\left(\xi_{2}-1\right)=0$, for every $1 \leq n \leq \min$ $\left\{M_{0}-1, M_{1}\right\}$. Using Lemma 4.4 we obtain that $k_{1}=k_{2}$ and also that $\xi_{1}=\xi_{2}$ in contradiction with the hypothesis of this Lemma.

Let us denote $\xi$ the common zero of $\mathrm{B}_{2}(x)$ and $\mathrm{A}_{3}(x)$. If $\mathrm{B}_{2}(\xi)=0$ we have $\Delta \mathrm{B}_{2}(\xi) \neq 0$, and hence $\mathrm{c}_{1}(\xi) \neq 0$. But we also obtain that $\mathrm{c}_{1}(\xi-1) \neq 0$, because if $\mathrm{c}_{1}(\xi-1)=0$ then it should be $\mathrm{B}_{2}(\xi-1)=\Delta \mathrm{B}_{2}(\xi-1)=0$ which is not possible.

From (4.1), setting $n=1$ and $x=\xi$, we obtain that $\pi(\xi)=0$.

THEOREM 4.6 Let $\left(u_{0}, u_{1}\right)$ be a $\Delta$-coherent pair of linear functionals, where $M_{0} \geq 2$ and $M_{1} \geq 4$ are the order of $u_{0}$ and $u_{1}$, respectively. Suppose that $\mathrm{B}_{2}(x)$ has not a double zero and also that no zero of $\mathrm{B}_{2}(x)$ is a root of $\Delta \mathrm{B}_{2}(x)=0$. Then 
(i) There exist a parameter $\xi$ and two polynomials $\tilde{A}(x)$ and $\pi_{1}(x)$ with $\operatorname{deg}(\tilde{A}) \leq 2$ and $\operatorname{deg}\left(\pi_{1}\right) \leq 1$ such that

$$
\begin{aligned}
\tilde{A}(x) u_{0} & =\frac{\sigma_{1} \sigma_{2}}{\left\langle u_{1}, \mathrm{~T}_{1}^{2}\right\rangle\left\langle u_{1}, \mathrm{~T}_{2}^{2}\right\rangle}(x-\xi) u_{1}, \\
\pi_{1}(x) u_{0} & =\frac{\sigma_{1} \sigma_{2}}{\left\langle u_{1}, \mathrm{~T}_{1}^{2}\right\rangle\left\langle u_{1}, \mathrm{~T}_{2}^{2}\right\rangle}(x-\xi) \Delta u_{1} .
\end{aligned}
$$

(ii) If $\tilde{A}(\xi)=0$ then $\pi_{1}(\xi)=0$.

(iii) The functional $u_{1}$ is a classical discrete linear functional verifying $\Delta\left[\tilde{A}(x+1) u_{1}\right]=$ $\tilde{\psi}_{1}(x) u_{1}$, where $\operatorname{deg}\left(\tilde{\psi}_{1}\right)=1$.

\section{Proof}

(i) Let us denote $\xi_{1}$ and $\xi_{2}$ the zeros of $\mathrm{B}_{2}(x)$. Using Lemma 4.5, at least one of them is also a zero of $\mathrm{A}_{3}(x)$. Suppose that $\mathrm{A}_{3}\left(\xi_{1}\right)=0$. Using again Lemma 4.5 we obtain that $\pi\left(\xi_{1}\right)=0$. Let us define

$$
\mathrm{B}_{2}(x)=\left(x-\xi_{1}\right) \tilde{B}(x), \quad \mathrm{A}_{3}(x)=\left(x-\xi_{1}\right) \tilde{A}(x), \quad \pi(x)=\left(x-\xi_{1}\right) \pi_{1}(x) .
$$

Then, we can divide both members of (4.1) by $x-\xi_{1}$ and we obtain

$$
\begin{gathered}
n \frac{\mathrm{P}_{n}(x)}{\left\langle u_{0}, \mathrm{P}_{n}^{2}\right\rangle} \tilde{B}(x)=\tilde{A}(x) \Delta \mathrm{c}_{n}(x-1)+\mathrm{c}_{n}(x-1) \pi_{1}(x), \\
1 \leq n \leq \min \left\{M_{0}-1, M_{1}\right\} .
\end{gathered}
$$

If we eliminate $u_{1}$ in (3.16) we get $\pi(x) u_{0}=\mathrm{B}_{2}(x) \Delta u_{1}$. From (4.15) we obtain

$$
\tilde{A}(x) u_{0}=\tilde{B}(x) u_{1}
$$

with an appropriate choice of the first moments of the functionals $u_{0}$ and $u_{1}$, which was to be proved. Furthermore, from (3.11) and (4.2) we get

$$
\pi_{1}(x) u_{0}=\tilde{B}(x) \Delta u_{1}+R \delta_{\xi_{1}}, \quad \tilde{A}(x) \Delta u_{1}=\pi_{1}(x) u_{1}+K \delta_{\xi_{1}} .
$$

Hence from (4.16) and (3.15) we obtain for $1 \leq n \leq \min \left\{M_{0}-1, M_{1}\right\}$

$$
\begin{aligned}
\left(\tilde{A}(x) \Delta \mathrm{c}_{n}(x-1)+\mathrm{c}_{n}(x-1) \pi_{1}(x)\right) u_{0} & =\left(n \tilde{B}(x) \frac{\mathrm{P}_{n}(x)}{\left\langle u_{0}, \mathrm{P}_{n}^{2}\right\rangle}\right) u_{0} \\
& =\tilde{B}(x)\left(\Delta \mathrm{c}_{n}(x-1) u_{1}+\mathrm{c}_{n}(x-1) \Delta u_{1}\right),
\end{aligned}
$$

i.e.,

$$
\begin{gathered}
\Delta \mathrm{c}_{n}(x-1)\left(\tilde{A}(x) u_{0}-\tilde{B}(x) u_{1}\right)=\mathrm{c}_{n}(x-1)\left(\tilde{B}(x) \Delta u_{1}-\pi_{1}(x) u_{0}\right), \\
1 \leq n \leq \min \left\{M_{0}-1, M_{1}\right\} .
\end{gathered}
$$

Moreover, using (4.17) the above identity becomes $\mathrm{c}_{n}(x-1) R \delta_{\xi_{1}}=0$ for every $1 \leq$ $n \leq \min \left\{M_{0}-1, M_{1}\right\}$. Since $\mathrm{c}_{1}\left(\xi_{1}-1\right) \neq 0$ we obtain $R=0$ and this proves (4.14). 
(ii) From the definition of $\tilde{A}(x)$ we have $\tilde{A}\left(\xi_{2}\right)=0$ and then, using Lemma 4.5 it follows that $\pi_{1}\left(\xi_{2}\right)=0$, so part (ii) of the Theorem is proved.

(iii) Finally, using (4.16) with $n=1$,

$$
\tilde{A}(x) \Delta \mathrm{c}_{1}(x-1)=\frac{\mathrm{P}_{1}(x)}{\left\langle u_{0}, \mathrm{P}_{1}^{2}\right\rangle} \tilde{B}(x)-\mathrm{c}_{1}(x-1) \pi_{1}(x)
$$

holds. If we take the first equation of (3.16) and the last equation it follows

$$
\begin{aligned}
\tilde{A}(x) \frac{\mathrm{P}_{1}(x)}{\left\langle u_{0}, \mathrm{P}_{1}^{2}\right\rangle} u_{0} & =\tilde{A}(x) \Delta \mathrm{c}_{1}(x-1) u_{1}+\tilde{A}(x) \mathrm{c}_{1}(x-1) \Delta u_{1} \\
& =\left(\frac{\mathrm{P}_{1}(x)}{\left\langle u_{0}, \mathrm{P}_{1}^{2}\right\rangle} \tilde{B}(x)-\mathrm{c}_{1}(x-1) \pi_{1}(x)\right) u_{1}+\tilde{A}(x) \mathrm{c}_{1}(x-1) \Delta u_{1},
\end{aligned}
$$

whence

$$
\frac{\mathrm{P}_{1}(x)}{\left\langle u_{0}, \mathrm{P}_{1}^{2}\right\rangle}\left(\tilde{A}(x) u_{0}-\tilde{B}(x) u_{1}\right)=\mathrm{c}_{1}(x-1)\left(\tilde{A}(x) \Delta u_{1}-\pi_{1}(x) u_{1}\right) .
$$

From the previous equation we obtain $K \mathrm{c}_{1}\left(\xi_{1}-1\right)=0$, by using (4.17) and (4.18). Since $c_{1}\left(\xi_{1}-1\right) \neq 0$, then $K=0$. Thus, the second equation in (4.18) reads as $\tilde{A}(x) \Delta u_{1}=\pi_{1}(x) u_{1}$. Therefore, by using Proposition 2.7 and this last equality, we get

$$
\Delta\left[\tilde{A}(x+1) u_{1}\right]=\Delta \tilde{A}(x) u_{1}+\tilde{A}(x) \Delta u_{1}=\left(\Delta \tilde{A}(x)+\pi_{1}(x)\right) u_{1}=\tilde{\psi}_{1}(x) u_{1},
$$

where $\operatorname{deg}\left(\tilde{\psi}_{1}\right) \leq 1$. As in Theorem 4.2 , we use that $u_{1}$ is weakly quasi-definite of order $M_{1} \geq 4$ to conclude $\operatorname{deg}\left(\tilde{\psi}_{1}\right)=1$, i.e., $u_{1}$ is a classical discrete linear functional.

Remark Note that if $u_{1}$ is a linear functional of order $M_{1}>7$, the polynomial $\mathrm{B}_{2}(x)$ can not have a double zero. For the proof, if $\xi$ is a double zero of $\mathrm{B}_{2}(x)$, then

$$
\mathrm{c}_{1}(x)=\frac{\sigma_{1}}{\left\langle u_{1}, \mathrm{~T}_{1}^{2}\right\rangle}\left(x-\left(\xi-\frac{1}{2}\right)\right)
$$

applying the $\Delta$ operator in the definition of $\mathrm{B}_{2}(x)$ given in (3.12). From (3.5) for $n=0$, it follows

$$
\frac{\phi_{0}(x)}{\left\langle u_{0}, \phi_{0}\right\rangle} u_{0}=-\mathrm{c}_{1}(x) u_{1}
$$

using (2.7) and the definition of $\mathrm{c}_{1}(x)$ in (3.10). We can now use the definition of $\phi_{0}(x)$ given in (3.13) as well as (3.11) in order to obtain

$$
\left(\frac{\phi_{1}(x) \mathrm{B}_{2}^{2}(x)}{\left\langle u_{0}, \phi_{0}\right\rangle}+\mathrm{c}_{1}(x)\right) u_{1}=0
$$


Since $u_{1}$ is a weakly quasi-definite linear functional of order greater than 7 , then

$$
\frac{\phi_{1}(x) \mathrm{B}_{2}^{2}(x)}{\left\langle u_{0}, \phi_{0}\right\rangle}+\mathrm{c}_{1}(x)=0 .
$$

By using (4.19) and since $\mathrm{B}_{2}(\xi)=0$, the above expression for $x=\xi$ gives $\sigma_{1}=0$ which is not possible.

We can summarize the results obtained in this section in the following theorem.

THEOREM 4.7 Let $\left(u_{0}, u_{1}\right)$ be a $\Delta$-coherent pair of linear functionals and let $\left\{\mathrm{P}_{n}(x)\right\}_{n=0}^{M_{0}}$ and $\left\{\mathrm{T}_{n}(x)\right\}_{n=0}^{M_{1}}$ be the corresponding MOPS associated with $u_{0}$ and $u_{1}$, respectively, with $M_{0} \geq 2$ and $M_{1} \geq 8$. Let

$$
\mathrm{B}_{2}(x):=\left|\begin{array}{cc}
\mathrm{c}_{1}(x-1) & \mathrm{c}_{2}(x-1) \\
\Delta \mathrm{c}_{1}(x-1) & \Delta \mathrm{c}_{2}(x-1)
\end{array}\right|=\frac{\sigma_{1} \sigma_{2}}{t_{1} t_{2}}(x-\xi)(x-\eta),
$$

where

$$
\mathrm{c}_{n}(x):=\sigma_{n} \frac{\mathrm{T}_{n}(x)}{t_{n}}-\frac{\mathrm{T}_{n} 1(x)}{t_{n 1}}, \quad t_{n} \quad:=\left\langle u_{1}, \mathrm{~T}_{n}^{2}{ }_{1}\right\rangle, \quad 1 \leq n \leq \min \left\{M_{0}-1, M_{1}\right\} .
$$

One of the following situations hold

(1) If $|\xi-\eta|=1$, then $u_{0}$ is a classical discrete linear functional satisfying

$$
\Delta\left[\tilde{\phi}(x-1) u_{0}\right]=\tilde{\psi}_{0}(x) u_{0}
$$

Moreover,

$$
\tilde{\phi}(x-1) u_{0}=\frac{\sigma_{1} \sigma_{2}}{t_{1} t_{2}}(x-\xi) u_{1} .
$$

(2) If $|\xi-\eta| \neq 1$, then $u_{1}$ is a classical discrete linear functional satisfying

$$
\Delta\left[\tilde{A}(x+1) u_{1}\right]=\tilde{\psi}_{1}(x) u_{1}
$$

Furthermore,

$$
\tilde{A}(x) u_{0}=\frac{\sigma_{1} \sigma_{2}}{t_{1} t_{2}}(x-\xi) u_{1}, \quad \pi_{1}(x) u_{0}=\frac{\sigma_{1} \sigma_{2}}{t_{1} t_{2}}(x-\xi) \Delta u_{1},
$$

where

$$
\pi_{1}(x):=\tilde{\psi}_{1}(x)-\Delta \tilde{A}(x)
$$

Finally, if $\tilde{A}(\xi)=0$ then $\pi_{1}(\xi)=0$.

From the above theorem, we have obtained in Ref. [5] the classification of all $\Delta$-coherent pairs for Hahn, Kravchuk, Meixner and Charlier linear functionals, which allowed us to recover the classification given by Meijer in Ref. [20], using a limit process. 


\section{EXAMPLES}

In this section we present examples of $\Delta$-coherent pairs $\left(u_{0}, u_{1}\right)$ of linear functionals. In the first example, we deal with quasi-definite linear functionals where $u_{0}$ or $u_{1}$ is the Meixner linear functional $u^{(\gamma, \mu)}$. On the other hand, in the second example we present $\Delta$-coherent pairs of linear functionals where $u_{0}$ or $u_{1}$ is the Hahn linear functional $u^{(\alpha, \beta, N)}$, which is a weakly quasi-definite linear functional of order $N-1$.

\subsection{Meixner Case}

Let $\left(u_{0}, u_{1}\right)$ be a $\Delta$-coherent pair of linear functionals and assume that $u_{1} \equiv u^{(\gamma, \mu)}$ is the Meixner linear functional defined by

$$
\left\langle u^{(\gamma, \mu)}, p\right\rangle=\sum_{s=0}^{\infty} p(s) \frac{\mu^{s} \Gamma(\gamma+s)(1-\mu)^{\gamma}}{\Gamma(s+1) \Gamma(\gamma)}, \quad 0<\mu<1, \quad \gamma>0, \text { for every } p \in \mathbb{P}
$$

[see Ref. 6 and references therein], which satisfies the distributional equation

$$
\Delta\left[\mu(x+\gamma) u^{(\gamma, \mu)}\right]=(\gamma \mu-x(1-\mu)) u^{(\gamma, \mu)} .
$$

For this quasi-definite linear functional the moments are given by

$$
\left(u^{(\gamma, \mu)}\right)_{n}=\sum_{m=0}^{n} \mathcal{S}_{m}(n)(\gamma)_{m}\left(\frac{\mu}{1-\mu}\right)^{m}, \quad n \geq 0
$$

where $\mathcal{S}_{m}(n)$ denotes the Stirling numbers of second kind [2]

$$
\mathcal{S}_{m}(n)=\sum_{j=0}^{m} \frac{(-1)^{m} j^{n}}{(m-j) ! j !}
$$

Let us denote by

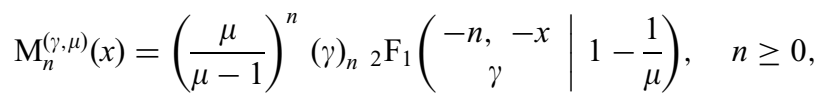

the polynomials orthogonal with respect to $u^{(\gamma, \mu)}$, which are called Meixner polynomials [see Ref. 10, p. 45, Ref. 21, p. 51]. The following relation between two families of Meixner polynomials holds [21, (2.4.16)]

$$
\mathrm{M}_{n}^{(\gamma+1, \mu)}(x)=\frac{\Delta \mathrm{M}_{n+1}^{(\gamma, \mu)}(x)}{n+1}, \quad n \geq 0 .
$$




\subsubsection{Case $u_{0} \equiv u^{(\gamma, \mu)}$}

Let $\left(u_{0}, u_{1}\right)$ be a $\Delta$-coherent pair of linear functionals and assume that $u_{0} \equiv u^{(\gamma, \mu)}$ is the Meixner linear functional. In this situation, the linear functional $u_{1}$ can be computed from (4.22)

$$
\mu(x+\gamma) u^{(\gamma, \mu)}=(x-\xi) u_{1} .
$$

By using Proposition 2.9 and (5.4), the above equation can be written as

$$
u^{(\gamma+1, \mu)}=(x-\xi) u_{1}
$$

Then, from (2.1) we get

$$
u_{1}=(x-\xi)^{1} u^{(\gamma+1, \mu)}+L \delta_{\xi}
$$

As an example, for $L=0$ and $\xi=0$ we shall obtain a recurrence relation for the sequences $\left\{\sigma_{n}\right\}$ and $\left\{\mathrm{T}_{n}(x)\right\}_{n=0}^{M_{1}}$, the MOPS associated with $u_{1}$ which can be computed from Ref. [8]

$$
\mathrm{T}_{n}(0) x \mathrm{M}_{n}^{(\gamma+1, \mu)}(x)=\mathrm{T}_{n}(0) \mathrm{T}_{n+1}(x)-\mathrm{T}_{n+1}(0) \mathrm{T}_{n}(x) .
$$

By using the above equation, (5.4), the three-term recurrence relation

$$
\left(x-\beta_{n}^{(\gamma+1, \mu)}\right) \mathbf{M}_{n}^{(\gamma+1, \mu)}(x)=\mathrm{M}_{n+1}^{(\gamma+1, \mu)}(x)+\gamma_{n}^{(\gamma+1, \mu)} \mathrm{M}_{n 1}^{(\gamma+1, \mu)}(x),
$$

satisfied by monic Meixner polynomials $\mathrm{M}_{n}^{(\gamma+1, \mu)}(x)$, where

$$
\begin{aligned}
& \beta_{n}^{(\gamma+1, \mu)}=\frac{(1+\gamma) \mu+(1+\mu) n}{1-\mu}, \\
& \gamma_{n}^{(\gamma+1, \mu)}=\frac{\mu n(\gamma+n)}{(1-\mu)^{2}},
\end{aligned}
$$

and the relation between $\left\{\mathrm{M}_{n}^{(\gamma+1, \mu)}(x)\right\}_{n}$ and $\left\{\mathrm{T}_{n}(x)\right\}_{n}$ since $\left(u_{0}, u_{1}\right)$ is a $\Delta$-coherent pair of linear functionals,

$$
\mathrm{T}_{n}(x)=\frac{\Delta \mathrm{M}_{n+1}^{(\gamma, \mu)}(x)}{n+1}-\sigma_{n} \frac{\Delta \mathrm{M}_{n}^{(\gamma, \mu)}(x)}{n}=\mathrm{M}_{n}^{(\gamma+1, \mu)}(x)-\sigma_{n} \mathrm{M}_{n 1}^{(\gamma+1, \mu)}(x), \quad n \geq 1,
$$

we obtain

$$
\left(\beta_{n}^{(\gamma+1, \mu)}+\sigma_{n+1}+\varpi_{n}\right) \mathbf{M}_{n}^{(\gamma+1, \mu)}(x)+\left(\gamma_{n}^{(\gamma+1, \mu)}-\sigma_{n} \varpi_{n}\right) \mathbf{M}_{n}^{(\gamma+1, \mu)}(x)=0,
$$

where

$$
\varpi_{n}=\frac{\mathrm{M}_{n+1}^{(\gamma+1, \mu)}(0)-\sigma_{n+1} \mathrm{M}_{n}^{(\gamma+1, \mu)}(0)}{\mathrm{M}_{n}^{(\gamma+1, \mu)}(0)-\sigma_{n} \mathrm{M}_{n}^{(\gamma+1, \mu)}(0)}
$$


and [21]

$$
\mathbf{M}_{n}^{(\gamma+1, \mu)}(0)=\left(\frac{\mu}{\mu-1}\right)^{n}(\gamma+1)_{n},
$$

being $(A)_{s}$ the Pochhammer symbol. Since $\left\{\mathrm{M}_{n}^{(\gamma+1, \mu)}(x)\right\}_{n=0}^{N}{ }^{2}$ is a set of linearly independent vectors in $\mathbb{P}$, it yields the following recurrence relation for the coefficients $\sigma_{n}$

$$
\sigma_{n+1}=-\beta_{n}^{(\gamma+1, \mu)}-\frac{\gamma_{n}^{(\gamma+1, \mu)}}{\sigma_{n}}, \quad n \geq 1,
$$

by using again the three-term recurrence relation (5.6).

If we choose $\left(u_{1}\right)_{0}:=\left\langle u_{1}, 1\right\rangle=1$, then $\mathrm{T}_{1}(x)=x-1$. From the $\Delta$-coherence relation,

$$
\sigma_{1}=1-\beta_{0}^{(\gamma+1, \mu)}
$$

Moreover, by using (5.6) and (5.8), from (5.7) we have

$$
\begin{aligned}
\mathrm{T}_{n}(x) & =\mathrm{M}_{n}^{(\gamma+1, \mu)}(x)+\left(\beta_{n 1}^{(\gamma+1, \mu)}+\frac{\gamma_{n}^{(\gamma+1, \mu)}}{\sigma_{n 1} 1}\right) \mathrm{M}_{n 1}^{(\gamma+1, \mu)}(x) \\
& =x \mathrm{M}_{n 1}^{(\gamma+1, \mu)}(x)+\gamma_{n 1}^{(\gamma+1, \mu)}\left(\frac{\mathrm{M}_{n 1}^{(\gamma+1, \mu)}(x)}{\sigma_{n} 1}-\mathrm{M}_{n 2}^{(\gamma+1, \mu)}(x)\right) \\
& =x \mathrm{M}_{n 1}^{(\gamma+1, \mu)}(x)+\frac{\gamma_{n}^{(\gamma+1, \mu)}}{\sigma_{n 1} 1} \mathrm{~T}_{n} 1(x), \quad n \geq 2 .
\end{aligned}
$$

a recurrence relation for the sequence $\left\{\mathrm{T}_{n}(x)\right\}_{n}$.

\subsubsection{Case $u_{1} \equiv u^{(\gamma, \mu)}$}

Let $\left(u_{0}, u_{1}\right)$ be a $\Delta$-coherent pair of linear functionals and assume that $u_{1} \equiv u^{(\gamma, \mu)}$ is the Meixner linear functional. We shall consider the following three situations:

Case $\gamma>1$ In this situation, from (4.23) we get

$$
\left.u_{0}=(x-\xi) u^{(\gamma} 1, \mu\right)
$$

We assume that $\xi \leq 0$ in order to obtain positive-definite linear functionals. We shall obtain a recurrence relation for the MOPS $\left\{\mathrm{P}_{n}(x)\right\}_{n}$ associated to $u_{0}$, as well as a recurrence relation for the coherence parameters $\sigma_{n}$.

The sequence $\left\{\mathrm{P}_{n}(x)\right\}_{n}$ can be computed from Ref. [8]

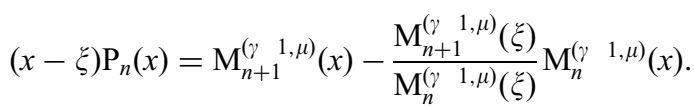

Moreover, they satisfy the following three-term recurrence relation

$$
\mathrm{P}_{n+1}(x)=\left(x-\tilde{B}_{n}\right) \mathrm{P}_{n}(x)-\tilde{C}_{n} \mathrm{P}_{n} 1(x), \quad n \geq 1, \quad \mathrm{P}_{0}(x):=1, \quad \mathrm{P}_{1}(x):=x-\tilde{B}_{0},
$$


where

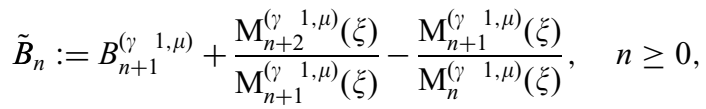

$$
\begin{aligned}
& \tilde{C}_{n}:=\frac{\mathrm{M}_{n}^{(\gamma \quad 1, \mu)}(\xi) \mathrm{M}_{n+1}^{(\gamma \quad 1, \mu)}(\xi)}{\left(\mathrm{M}_{n}^{(\gamma 1, \mu)}(\xi)\right)^{2}} C_{n}^{(\gamma \quad 1, \mu)}, \quad n \geq 1,
\end{aligned}
$$

and

$$
B_{n}^{(\gamma \quad 1, \mu)}=\frac{(\gamma-1) \mu+n(1+\mu)}{1-\mu}, \quad C_{n}^{(\gamma \quad 1, \mu)}=\frac{\mu n(\gamma+n-2)}{(1-\mu)^{2}},
$$

are the coefficients of the three-term recurrence relation satisfied by monic Meixner polynomials $\mathbf{M}_{n}^{(\gamma}{ }^{1, \mu)}(x)$

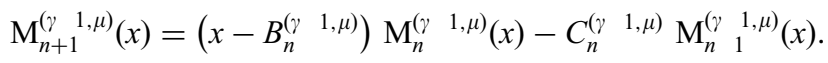

By using

$$
\mathrm{M}_{n}^{(\gamma, \mu)}(x)=\frac{\Delta \mathrm{P}_{n+1}(x)}{n+1}-\sigma_{n} \frac{\Delta \mathrm{P}_{n}(x)}{n}, \quad n \geq 1
$$

as well as (5.9), (5.10) and (5.11), we obtain

$$
\sigma_{n}=\xi-\tilde{B}_{n}-\frac{\tilde{C}_{n}}{\sigma_{n 1}}, \quad n \geq 2
$$

with

$$
\sigma_{1}:=\frac{\gamma \mu(-\xi+\mu(-1+\gamma+\xi))}{(-1+\mu)\left(\gamma^{2} \mu^{2}+(-1+\mu)^{2}(-1+\xi) \xi+\gamma \mu(-\mu+2(-1+\mu) \xi)\right)} .
$$

Case $\gamma=1$ Then, up to numerical factors, $u_{0}$ can be deduced from (4.23)

$$
x u_{0}=(x-\xi) u^{(1, \mu)} .
$$

From Lemma $4.5, \xi=0$ and

$$
u_{0}=u^{(1, \mu)}+K \delta_{0}
$$

using (2.1). Let $K>0$ and let us denote by $\left\{\mathrm{P}_{n}(x)\right\}_{n=0}^{\infty}$ the sequence of polynomials orthogonal with respect to this quasi-definite linear functional $u_{0}$ (perturbation of $u^{(1, \mu)}$ by a Dirac functional). These polynomials satisfy the following three-term recurrence relation [13]

$$
\begin{aligned}
\mathrm{P}_{n+1}(x) & =\left(x-\tilde{B}_{n}\right) \mathrm{P}_{n}(x)-\tilde{C}_{n} \mathrm{P}_{n}(x), \quad n \geq 1, \\
\mathrm{P}_{0}(x) & =1, \quad \mathrm{P}_{1}(x)=x-\tilde{B}_{0},
\end{aligned}
$$


where

$$
\begin{aligned}
& \tilde{B}_{n}=-n-\frac{2 n+1}{\mu-1}+\frac{n(K+1)}{1+K\left(1-\mu^{n}\right)}+\frac{(n+1)(K+1)}{K\left(\mu^{n+1}-1\right)-1}, \quad n \geq 0, \\
& \tilde{C}_{n}=\frac{n^{2} \mu\left(K\left(\mu^{n} 1-1\right)-1\right)\left(K\left(\mu^{n+1}-1\right)-1\right)}{(1-\mu)^{2}\left(K\left(\mu^{n}-1\right)-1\right)^{2}}, \quad n \geq 1 .
\end{aligned}
$$

Furthermore, they can be written in terms of hypergeometric series [4]

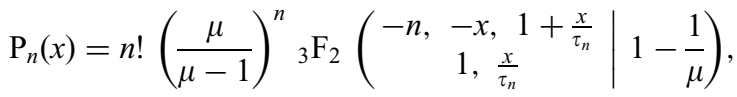

where

$$
\tau_{n}=\frac{K \mu^{n}}{1+K\left(1-\mu^{n}\right)}, \quad K>0
$$

It can be checked that the $\Delta$-coherence relation reads

$$
\mathrm{M}_{n}^{(1, \mu)}(x)=\frac{\Delta \mathrm{P}_{n+1}(x)}{n+1}-\sigma_{n} \frac{\Delta \mathrm{P}_{n}(x)}{n}, \quad n \geq 1,
$$

where

$$
\sigma_{n}=n\left(\frac{1}{\mu-1}+\frac{K+1}{1+K\left(1-\mu^{n+1}\right)}\right), \quad n \geq 1
$$

are non-zero real numbers, since $K>0$.

Case $0<\gamma<1 \quad$ In this situation $u_{0}=u_{1}=u^{(\gamma, \mu)}$. The coherence relation reads

$$
\mathrm{M}_{n}^{(\gamma, \mu)}(x)=\frac{\Delta \mathrm{M}_{n+1}^{(\gamma, \mu)}(x)}{n+1}-\sigma_{n} \frac{\Delta \mathrm{M}_{n}^{(\gamma, \mu)}(x)}{n}, \quad n \geq 1,
$$

where

$$
\sigma_{n}=n \frac{\mu}{\mu-1}
$$

\subsection{Hahn Case}

Let $u^{(\alpha, \beta, N)}$ be the Hahn linear functional given by

$$
\left\langle u^{(\alpha, \beta, N)}, r\right\rangle=\sum_{s=0}^{N} \frac{\Gamma(N) \Gamma(\alpha+\beta+2) \Gamma(\alpha+N-s) \Gamma(\beta+s+1)}{\Gamma(\alpha+1) \Gamma(\beta+1) \Gamma(\alpha+\beta+N+1) \Gamma(N-s) \Gamma(s+1)} r(s),
$$

for every $r \in \mathbb{P}$, 
where $\alpha>-1, \beta>-1$, and $N \in \mathbb{N}$, which satisfies the distributional equation

$$
\Delta\left[(N-x-1)(x+\beta+1) u^{(\alpha, \beta, N)}\right]=((N-1)(\beta+1)-x(\alpha+\beta+2)) u^{(\alpha, \beta, N)} .
$$

For this weakly quasi-definite linear functional of order $N-1$ the moments are given by

$$
\left(u^{(\alpha, \beta, N)}\right)_{n}=\sum_{m=0}^{n} \mathcal{S}_{m}(n)(-1)^{m} \frac{(1-N)_{m}(\beta+1)_{m}}{(\alpha+\beta+2)_{m}}, \quad n \geq 0,
$$

where $(A)_{n}$ denotes the Pochhammer symbol and $\mathcal{S}_{m}(n)$ are the Stirling numbers of second kind given in (5.3).

Let us denote by

$$
\begin{gathered}
\mathrm{h}_{n}^{(\alpha, \beta)}(x ; N)=\frac{(1)^{n}(N n)_{n}(\beta+1)_{n}}{(\alpha+\beta+n+1)_{n}}{ }_{3} \mathrm{~F}_{2}\left(\begin{array}{c|c}
-n, & -x, n+\alpha+\beta+1 \\
\beta+1,1-N & 1
\end{array}\right), \\
0 \leq n \leq N-1,
\end{gathered}
$$

the polynomials orthogonal with respect to $u^{(\alpha, \beta, N)}$, which are called the Hahn polynomials [see Ref. 10, p. 33, Ref. 21, p. 52]. The following relation between two families of Hahn polynomials holds [21, (2.4.13)]

$$
\mathrm{H}_{n}^{(\alpha+1, \beta+1)}(x ; N-1)=\frac{\Delta \mathrm{H}_{n+1}^{(\alpha, \beta)}(x ; N)}{n+1}, \quad 0 \leq n \leq N-2 .
$$

\subsubsection{Case $u_{0} \equiv u^{(\alpha, \beta, N)}$}

Let $\left(u_{0}, u_{1}\right)$ be a $\Delta$-coherent pair of linear functionals and assume that $u_{0} \equiv u^{(\alpha, \beta, N)}$ is the Hahn linear functional. In this situation, the linear functional $u_{1}$ can be computed from (4.22)

$$
(N-x-1)(x+\beta+1) u^{(\alpha, \beta, N)}=(x-\xi) u_{1} .
$$

By using Proposition 2.9 and (5.12), the above equation can be written as

$$
\left.u^{(\alpha+1, \beta+1, N} 1\right)=(x-\xi) u_{1} .
$$

Then, from (2.1) we get

$$
\left.u_{1}=(x-\xi){ }^{1} u^{(\alpha+1, \beta+1, N} 1\right)+L \delta_{\xi} .
$$

If $L=0$ and $\xi=0$, we shall obtain a recurrence relation for the sequences $\left\{\sigma_{n}\right\}$ and $\left\{\mathrm{T}_{n}(x)\right\}_{n=0}^{M_{1}}$, the MOPS associated with $u_{1}$ which can be computed from [8]

$$
\mathrm{T}_{n}(0) x \mathrm{H}_{n}^{(\alpha+1, \beta+1)}(x ; N-1)=\mathrm{T}_{n}(0) \mathrm{T}_{n+1}(x)-\mathrm{T}_{n+1}(0) \mathrm{T}_{n}(x) .
$$

By using the above equation, (5.12), the three-term recurrence relation

$$
\begin{aligned}
\left(x-\beta_{n}^{(\alpha+1, \beta+1, N 1)}\right) \mathrm{H}_{n}^{(\alpha+1, \beta+1)}(x ; N-1)= & \left.\mathrm{H}_{n+1}^{(\alpha+1, \beta+1)}(x ; N-1)+\gamma_{n}^{(\alpha+1, \beta+1, N} 1\right) \\
& \times \mathrm{H}_{n 1}^{(\alpha+1, \beta+1)}(x ; N-1),
\end{aligned}
$$


satisfied by monic Hahn polynomials $\mathrm{H}_{n}^{(\alpha+1, \beta+1)}(x ; N-1)$, where

$$
\begin{aligned}
& \left.\beta_{n}^{(\alpha+1, \beta+1, N} 1\right) \\
& =\frac{n^{2}(2 N+\alpha-\beta-4)+(N-2)(\beta+2)(\alpha+\beta+2)+n(2 N+\alpha-\beta-4)(\alpha+\beta+3)}{(2 n+\alpha+\beta+2)(2 n+\alpha+\beta+4)}, \\
& \left.\gamma_{n}^{(\alpha+1, \beta+1, N} 1\right) \\
& =\frac{n(N-n-1)(n+\alpha+1)(n+\beta+1)(n+\alpha+\beta+2)(n+N+\alpha+\beta+1)}{(2 n+\alpha+\beta+1)(2 n+\alpha+\beta+2)^{2}(2 n+\alpha+\beta+3)},
\end{aligned}
$$

and the relation between $\left\{\mathrm{H}_{n}^{(\alpha+1, \beta+1)}(x ; N-1)\right\}_{n=0}^{N} 2$ and $\left\{\mathrm{T}_{n}(x)\right\}_{n=0}^{M_{1}}$ since $\left(u_{0}, u_{1}\right)$ is a $\Delta$-coherent pair of linear functionals,

$$
\begin{gathered}
\mathrm{T}_{n}(x)=\frac{\Delta \mathrm{H}_{n+1}^{(\alpha, \beta)}(x ; N)}{n+1}-\sigma_{n} \frac{\Delta \mathrm{H}_{n}^{(\alpha, \beta)}(x ; N)}{n}=\mathrm{H}_{n}^{(\alpha+1, \beta+1)}(x ; N-1) \\
-\sigma_{n} \mathrm{H}_{n 1}^{(\alpha+1, \beta+1)}(x ; N-1), 1 \leq n \leq \min \left\{N-2, M_{1}\right\},
\end{gathered}
$$

we obtain

$$
\begin{aligned}
& \left.\left(\beta_{n}^{(\alpha+1, \beta+1, N} 1\right)+\sigma_{n+1}+\varpi_{n}\right) \mathrm{H}_{n}^{(\alpha+1, \beta+1)}(x ; N-1) \\
& \left.+\left(\gamma_{n}^{(\alpha+1, \beta+1, N} 1\right)-\sigma_{n} \varpi_{n}\right) \mathrm{H}_{n}^{(\alpha+1, \beta+1)}(x ; N-1)=0,
\end{aligned}
$$

where

$$
\varpi_{n}=\frac{\mathrm{H}_{n+1}^{(\alpha+1, \beta+1)}(0 ; N-1)-\sigma_{n+1} \mathrm{H}_{n}^{(\alpha+1, \beta+1)}(0 ; N-1)}{\mathrm{H}_{n}^{(\alpha+1, \beta+1)}(0 ; N-1)-\sigma_{n} \mathrm{H}_{n}^{(\alpha+1, \beta+1)}(0 ; N-1)},
$$

and [21]

$$
\mathrm{H}_{n}^{(\alpha+1, \beta+1)}(0 ; N-1)=(-1)^{n} \frac{(\beta+2)_{n}(N-n-1)_{n}}{(n+\alpha+\beta+3)_{n}},
$$

being $(A)_{s}$ the Pochhammer symbol. Since $\left\{\mathrm{H}_{n}^{(\alpha+1, \beta+1)}(x ; N-1)\right\}_{n=0}^{N} 2$ is a set of linearly independent vectors in $\mathbb{P}_{N}$, it yields the following recurrence relation for the coefficients $\sigma_{n}$

$$
\left.\sigma_{n+1}=-\beta_{n}^{(\alpha+1, \beta+1, N} 1\right)-\frac{\left.\gamma_{n}^{(\alpha+1, \beta+1, N} 1\right)}{\sigma_{n}}, \quad n \geq 1,
$$

by using again the three-term recurrence relation (5.14).

If we choose $\left(u_{1}\right)_{0}:=\left\langle u_{1}, 1\right\rangle=1$, then $\mathrm{T}_{1}(x)=x-1$. From the $\Delta$-coherence relation,

$$
\left.\sigma_{1}=1-\beta_{0}^{(\alpha+1, \beta+1, N} 1\right)
$$


Moreover, by using (5.14) and (5.16), from (5.15) we have

$$
\begin{aligned}
& \mathrm{T}_{n}(x)=\mathrm{H}_{n}^{(\alpha+1, \beta+1)}(x ; N-1)+\left(\begin{array}{cc}
\left.\beta_{n}^{(\alpha+1, \beta+1, N} 1\right) & 1 \frac{\left.\gamma_{n}^{(\alpha+1, \beta+1, N} 1\right)}{\sigma_{n} 1}
\end{array}\right) \mathrm{H}_{n}^{(\alpha+1, \beta+1)}(x ; N-1) \\
& \left.=x \mathrm{H}_{n 1}^{(\alpha+1, \beta+1)}(x ; N-1)+\gamma_{n 1}^{(\alpha+1, \beta+1, N} 1\right) \\
& \times\left(\frac{\mathrm{H}_{n 1}^{(\alpha+1, \beta+1)}(x ; N-1)}{\sigma_{n 1}}-\mathrm{H}_{n 2}^{(\alpha+1, \beta+1)}(x ; N-1)\right) \\
& =x \mathrm{H}_{n 1}^{(\alpha+1, \beta+1)}(x ; N-1)+\frac{\left.\gamma_{n}^{(\alpha+1, \beta+1, N} 1\right)}{\sigma_{n} 1} \mathrm{~T}_{n} \quad 1(x), \quad n \geq 2 .
\end{aligned}
$$

a recurrence relation for the sequence $\left\{\mathrm{T}_{n}(x)\right\}_{n=0}^{M_{1}}$.

\subsubsection{Case $u_{1} \equiv u^{(\alpha, \beta, N)}$}

Let $\left(u_{0}, u_{1}\right)$ be a $\Delta$-coherent pair of linear functionals and assume that $u_{1} \equiv u^{(\alpha, \beta, N)}$ is the Hahn linear functional. We must consider the following situations:

Case $\alpha, \beta>0$ In this situation, from (4.23) we get

$$
\left.u_{0}=(x-\xi) u^{(\alpha} \quad 1, \beta \quad 1, N+1\right) .
$$

We assume that $\xi \leq 0$ or $\xi \geq N$ in order to obtain nonnegative-definite linear functionals. We shall obtain a recurrence relation for the MOPS $\left\{\mathrm{P}_{n}(x)\right\}_{n=0}^{M_{0}}$ associated to $u_{0}$, as well as a recurrence relation for the coherence parameters $\sigma_{n}$.

The monic sequence $\left\{\mathrm{P}_{n}(x)\right\}_{n=0}^{M_{0}}$ can be computed from [8]

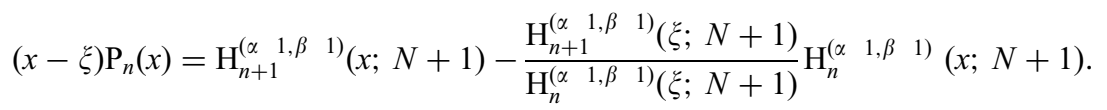

Moreover, they satisfy the following three-term recurrence relation

$$
\mathrm{P}_{n+1}(x)=\left(x-\tilde{B}_{n}\right) \mathrm{P}_{n}(x)-\tilde{C}_{n} \mathrm{P}_{n} \quad 1(x), \quad 1 \leq n \leq M_{0}-1, \quad \mathrm{P}_{0}(x):=1, \quad \mathrm{P}_{1}(x):=x-\tilde{B}_{0},
$$

where

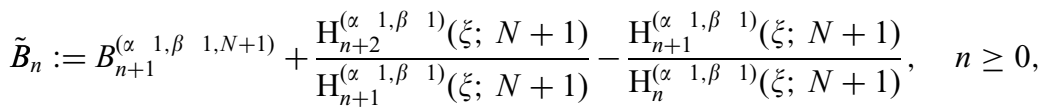

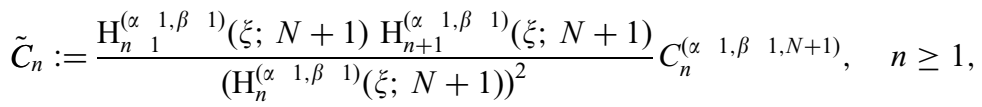


and

$$
\begin{aligned}
& \left.B_{n}^{(\alpha} \quad 1, \beta \quad 1, N+1\right)=\frac{\beta(-2+\alpha+\beta) N+n(-1+\alpha+\beta+n)(\alpha-\beta+2 N)}{(-2+\alpha+\beta+2 n)(\alpha+\beta+2 n)} \\
\left.C_{n}^{(\alpha \quad 1, \beta} \quad 1, N+1\right) & \\
= & -\frac{n(\alpha+n-1)(\beta+n-1)(\alpha+\beta+n-2)(n-N-1)(\alpha+\beta+n+N-1)}{(\alpha+\beta+2 n-3)(-2+\alpha+\beta+2 n)^{2}(-1+\alpha+\beta+2 n)},
\end{aligned}
$$

are the coefficients of the three-term recurrence relation

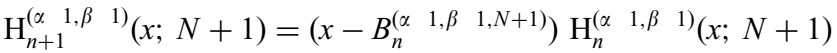

$$
\begin{aligned}
& -C_{n}^{(\alpha 1, \beta}{ }^{1, N+1)} \mathrm{H}_{n}^{(\alpha \quad 1, \beta}{ }^{1)}(x ; N+1) .
\end{aligned}
$$

satisfied by monic Hahn polynomials $\mathrm{H}_{n}^{(\alpha} \quad{ }^{1, \beta} \quad{ }^{1)}(x ; N+1)$. By using

$$
\mathrm{H}_{n}^{(\alpha, \beta)}(x ; N)=\frac{\Delta \mathrm{P}_{n+1}(x)}{n+1}-\sigma_{n} \frac{\Delta \mathrm{P}_{n}(x)}{n}, \quad n \geq 1,
$$

as well as (5.17), (5.18) and (5.19), we obtain

$$
\sigma_{n}=\xi-\tilde{B}_{n}-\frac{\tilde{C}_{n}}{\sigma_{n 1}}, \quad n \geq 2,
$$

with

$$
\sigma_{1}:=\frac{(1+\alpha)(1+\beta)(N-1)(\vartheta+N)((\alpha+\beta)-\beta N \xi)}{\left.(1+\vartheta)(2+\vartheta)(\beta(1+\beta)(N-1) N-\vartheta(\alpha-\beta+2(1+\beta) N) \xi+\vartheta)(1+\vartheta) \xi^{2}\right)},
$$

and $\vartheta:=1+\alpha+\beta$.

Case $\beta=0 \quad$ From Lemma 4.5 then $\xi=0$, and from (4.23)

$$
u_{0}=u^{(\alpha 1,0, N+1)}+L \delta_{0}, \quad \alpha>0 .
$$

Let us assume that $L \geq 0$ and let us denote by $\left\{\mathrm{P}_{n}(x)\right\}_{n=0}^{N}$ the sequence of polynomials orthogonal with respect to this weakly quasi-definite linear functional $u_{0}$ (perturbation of $u^{(\alpha 1,0, N+1)}$ by a Dirac functional). These polynomials can be written in terms of hypergeometric series [3]

$$
\mathrm{P}_{n}(x)=\frac{(-1)^{n}(N) ! n ! \Gamma(\alpha+n)}{(N-n) ! \Gamma(\alpha+2 n)}{ }_{4} \mathrm{~F}_{3}\left(\begin{array}{c}
-n,-x, \alpha+n-1, \eta_{0}+1 \\
-N, 1, \eta_{0}
\end{array} \mid 1\right), \quad 0 \leq n \leq N,
$$


where

$$
\begin{aligned}
\eta_{0} & :=\frac{x(\alpha+n-1)}{x+(\alpha+2 n-1) \tau_{n}}, \\
\tau_{n} & :=\frac{L}{\left(1+\operatorname{Ler}_{n}^{(\alpha 11,0)}(0,0)\right)} \frac{(1+\alpha)_{2(n}{ }_{1}(N-n+1)_{n}}{(\alpha)_{n} 1^{1}(\alpha+n)_{n}(1+\alpha+N)_{n} 1}, \\
\operatorname{Ker}_{n}^{(\alpha \quad 1,0)}(0,0) & :=\sum_{m=0}^{n} \frac{(-1)^{m}(1+\alpha)_{2 m}\left((N-m+1)_{m}\right)^{2}}{(\alpha)_{m}(\alpha+m)_{m}(-N)_{m}(1+\alpha+N)_{m}} .
\end{aligned}
$$

It can be checked that the $\Delta$-coherence relation reads

$$
\mathrm{H}_{n}^{(\alpha, 0)}(x ; N)=\frac{\Delta \mathrm{P}_{n+1}(x)}{n+1}-\sigma_{n} \frac{\Delta \mathrm{P}_{n}(x)}{n}, \quad 1 \leq n \leq N-1,
$$

where,

$$
\sigma_{n}=\frac{-n(\alpha+n)(N-n)(\alpha+N+n)\left((\alpha+N+1)_{n}(L N+\alpha(L+1))-L(N-n+1)_{n}\right)}{(\alpha+2 n)(\alpha+2 n+1)\left((\alpha+N+1)_{n}(L N+\alpha(L+1))-L(N-n)_{n+1}\right)}
$$

are non-zero real numbers.

Remark 3 Note that the classification of $\Delta$-coherent pairs, assuming that one of the linear functionals $u_{0}$ or $u_{1}$ is the Charlier or the Kravchuk linear functional, can be done by using the same arguments as in the previous examples [5].

\section{Acknowledgements}

F. Marcellán wishes to acknowledge Dirección General de Investigación (MCYT) of Spain for financial support under grant BFM2000-0206C04-01 and INTAS project 2000-272.

\section{References}

[1] Abdelkarim, F. and Maroni, P. (1997). The $D_{\omega}$-classical orthogonal polynomials. Result. Math., 32, 128.

[2] Abramowitz, M. and Stegun, I. (1965). Handbook of Mathematical Functions. Dover, New York.

[3] Álvarez-Nodarse, R. and Marcellán, F. (1995). The modification of classical Hahn polynomials of a discrete variable. Integral Transforms and Special Functions, 4(3), 243262.

[4] Álvarez-Nodarse, R., García, A. G. and Marcellán, F. (1995). On the properties for modifications of classical orthogonal polynomials of discrete variables. J. Comput. Appl. Math., 65, 318.

[5] Area, I., Godoy, E. and Marcellán, F. (2000). Classification of all $\Delta$-coherent pairs. Integral Transforms and Special Functions, 9(1), 118.

[6] Chihara, T. S. (1978). An Introduction to Orthogonal Polynomials. Gordon and Breach, New York.

[7] García, A. G., Marcellán, F. and Salto, L. (1995). A distributional study of discrete classical orthogonal polynomials. J. Comput. Appl. Math., 57, 147162.

[8] Gautschi, W. (1982). An algorithmic implementation of the generalized Christoffel theorem. In: Hämmerlin, G. (Ed.), Numerical Integration. Proceedings of the Conference 1981 Held at the Mathematisches Forschungsinstitut Oberwolfach, October 4 10, International Series of Numerical Mathematics, Vol. 57. Basel Boston Stuttgart, Birkhäuser Verlag, pp. 89106.

[9] Iserles, A., Koch, P. E., Nørsett, S. P. and Sanz-Serna, J. M. (1991). On Polynomials orthogonal with respect to certain Sobolev Inner Products. J. Approx. Theory, 65, 151175.

[10] Koekoek, R. and Swarttouw, R. F. (1998). The Askey-scheme of hypergeometric orthogonal polynomials and its q-analogue, Report 98 17, Fac. Techn. Math. Informatics, Delft University of Technology, Delft. 
[11] Lesky, P. A. (1995). Vervollständigung der klassischen Orthogonalpolynome durch Ergänzungen zum AskeySchema der hypergeometrischen orthogonalen Polynome. Sitzungsber. Abt. II, 204, 151166.

[12] Levenshtein, V. I. (1995). Krawtchouk polynomials and universal bounds for codes and design in Hamming spaces. IEEE Trans. Inform. Theory, 41,1303 1321.

[13] Marcellán, F. and Maroni, P. (1992). Sur l'adjonction d'une masse de Dirac á une forme régulière et semiclassique. Ann. Mat. Pura ed Appl. (IV), CLXII, 122.

[14] Marcellán, F., Martínez-Finkelshtein, A. and Moreno-Balcazar, J. J. (1997). Asymptotics of Sobolev orthogonal polynomials for symmetrically coherent pairs of measures with compact support. J. Comput. Appl. Math., 81(2), 217227.

[15] Marcellán, F. and Petronilho, J. C. (1995). Orthogonal polynomials and coherent pairs: the classical case. Indag. Mathem. N.S., 6(3), 287307.

[16] Marcellán, F. and Petronilho, J. C., Pérez, T. E. and Piñar, M. A. (1995). What is beyond coherent pairs of orthogonal polynomials?. J. Comput. Appl. Math., 65, 267277.

[17] Marcellán, F. and Salto, L. (1998). Discrete semi-classical orthogonal polynomials. J. Difference Equations, 4, 463496.

[18] Maroni, P. (1991). Une théorie algébrique des polynômes orthogonaux. Application aux polynômes orthogonaux semi-classiques. In: Brezinski, C., Gori, L. and Ronveaux, A. (Eds.), Orthogonal Polynomials and Their Applications, Vol. 9. Basel, Switzerland, J. C. Baltzer A. G, pp. 95130.

[19] Martínez-Finkelshtein, A., Moreno Balcázar, J. J., Pérez, T. E. and Piñar, M. A. (1998). Asymptotics of Sobolev orthogonal polynomials for coherent pairs of measures. J. Approx. Theory, 92(2), 280293.

[20] Meijer, H. G. (1997). Determination of all coherent pairs. J. Approx. Theory, 89, 321343.

[21] Nikiforov, A. F., Suslov, S. K. and Uvarov, V. B. (1991). Classical Orthogonal Polynomials of a Discrete Variable. Springer Verlag, Berlin.

[22] Pan, K. (1998). Asymptotics for Sobolev orthogonal polynomials with coherent pairs: The Jacobi case, type I. Proc. Amer. Math. Soc., 126, 23772388.

[23] Pan, K. (1997). On Sobolev orthogonal polynomials with coherent pairs. The Jacobi case. J. Comput Appl. Math., 79(2), 249262. 